\title{
Inhibition of Wnt/ $\beta$-catenin signaling ameliorates osteoarthritis in a murine model of experimental osteoarthritis
}

\author{
Caressa Lietman, ${ }^{1}$ Brian Wu, ${ }^{2}$ Sarah Lechner, ${ }^{1}$ Andrew Shinar, ${ }^{3}$ Madhur Sehgal, ${ }^{4}$ \\ Evgeny Rossomacha, ${ }^{4}$ Poulami Datta, ${ }^{4}$ Anirudh Sharma, ${ }^{4}$ Rajiv Gandhi, ${ }^{2}$ Mohit Kapoor, ${ }^{2,4}$ \\ and Pampee P. Young ${ }^{1,5,6}$
}

'Department of Pathology, Microbiology and Immunology, Vanderbilt University Medical Center, Nashville, Tennessee, USA. ${ }^{2}$ Arthritis Program, University Health Network, Toronto, Ontario, Canada. ${ }^{3}$ Orthopedic Institute, Vanderbilt University Medical Center, Nashville, Tennessee, USA. ${ }^{4}$ Department of Surgery and Department of Laboratory Medicine and Pathobiology, University of Toronto, and Division of Genetics and Development, Krembil Research Institute, University Health Network, Toronto, Ontario, Canada. ${ }^{5}$ Department of Cell and Developmental Biology and Department of Internal Medicine, Vanderbilt University Medical Center, Nashville, Tennessee, USA. ${ }^{6}$ Department of Veterans Affairs Medical Center, Nashville, Tennessee, USA.

\begin{abstract}
Osteoarthritis (OA) is a degenerative joint disease involving both cartilage and synovium. The canonical Wnt/ $\beta$-catenin pathway, which is activated in $\mathrm{OA}$, is emerging as an important regulator of tissue repair and fibrosis. This study seeks to examine Wnt pathway effects on synovial fibroblasts and articular chondrocytes as well as the therapeutic effects of Wnt inhibition on OA disease severity. Mice underwent destabilization of the medial meniscus surgery and were treated by intra-articular injection with XAV-939, a small-molecule inhibitor of Wnt/ $\beta$-catenin signaling. $W n t / \beta$-catenin signaling was highly activated in murine synovial fibroblasts as well as in OAderived human synovial fibroblasts. XAV-939 ameliorated OA severity associated with reduced cartilage degeneration and synovitis in vivo. Wnt inhibition using mechanistically distinct smallmolecule inhibitors, XAV-939 and C113, attenuated the proliferation and type I collagen synthesis in synovial fibroblasts in vitro but did not affect human $\mathrm{OA}$-derived chondrocyte proliferation. However, Wnt modulation increased COL2A1 and PRG4 transcripts, which are downregulated in chondrocytes in OA. In conclusion, therapeutic Wnt inhibition reduced disease severity in a model of traumatic $\mathrm{OA}$ via promoting anticatabolic effects on chondrocytes and antifibrotic effects on synovial fibroblasts and may be a promising class of drugs for the treatment of $\mathrm{OA}$.
\end{abstract}

Authorship note: C. Lietman and B. Wu are co-first authors.

Conflict of interest: The authors have declared that no conflict of interest exists.

Submitted: July 21, 2017 Accepted: December 12, 2017 Published: January 25, 2018

Reference information: JCI Insight. 2018;3(2):e96308. https://doi.org/10.1172/jci. insight. 96308.

\section{Introduction}

Osteoarthritis (OA) is a degenerative joint disease typically characterized by articular cartilage degeneration, bone remodeling, and osteophytosis as well as fibrosis and hyperplasia of the synovial membrane (1). Although the synovium is an important player in OA pathology, synovial contribution toward OA development and progression is less well understood (2). While OA is highly prevalent and associated with significant morbidity, there remains a lack of effective pharmacologic treatment $(3,4)$. Targeting detrimental signaling pathways within cartilage and synovium may holistically decelerate total joint destruction at earlier stages of OA to retard disease progression.

Wnt family proteins are a class of morphogens associated with embryonic skeletal formation, tissue repair, fibrosis, and joint homeostasis $(5,6)$. Wnts regulate multiple signaling cascades, including the $\beta$-catenin-dependent (canonical) pathway (5, 7-9). The Wnt/ $\beta$-catenin pathway, which is typically quiescent in many adult organs, is activated in response to injury (10-12). Its role in tissue repair and regeneration is complex and incompletely understood, although an increasing body of data suggests that its activation augments fibrotic repair (13). Our group recently published studies demonstrating that brief therapeutic Wnt inhibition following both full thickness cutaneous or ischemic cardiac injury resulted in improved regenerative repair with less fibrosis $(14,15)$. 
In OA pathogenesis, activation of canonical Wnt signaling is observed in both articular cartilage and synovium following injury, with increased expression of both Wnt ligands and target genes (16, 17). Induced overexpression of $\beta$-catenin within mature chondrocytes has been shown to exacerbate cartilage degeneration, chondrocyte hypertrophy, and expression of matrix proteases $(6,18-20)$. However, significant or complete ablation of $\beta$-catenin in chondrocytes also results in the deleterious effect of chondrocyte apoptosis (21). Moreover, canonical Wnt expression in the synovium mediated by adenoviral transduction with Wnt ligands resulted in strong induction of cartilage pathology (22). Less is known about the effect of Wnt inhibition, with contradictory findings using overexpression or inhibition of Dickkopf-related protein $1(D k k 1)(23,24)$. Although the sum of the published data using genetic modulation of Wnt suggests deleterious effects of Wnt on OA pathogenesis, there is little published on the cellular target(s) of Wnt modulation and specific cellular phenotypes induced by Wnt modulation, particularly within the synovium $(18,25)$. There is also little known about the therapeutic effect of inhibiting the Wnt pathway within the context of disease.

Multiple Wnt inhibitory therapeutics are being investigated at various stages of clinical development due to Wnt pathway activation, not only in fibrotic diseases, but also cancers (26-29). We sought to use known Wnt inhibitors that specifically target the canonical Wnt pathway. One well-characterized small-molecule inhibitor, XAV-939, is commercially available; it acts as a tankyrase inhibitor to stabilize AXIN2, and thereby the destruction complex, and promotes the phosphorylation-dependent degradation of $\beta$-catenin (30). In luciferase reporter assays, it did not affect cAMP response elements, NF- $\kappa B$ or TGF- $\beta$ pathways (30). Another well-characterized small-molecule inhibitor, pyrvinium, and its structural analog, VU-WS113 (C113), activate casein kinase $1 \alpha$, thereby enabling degradation of $\beta$-catenin without affecting BMP4, TGF- $\alpha$, IL-4, or Notch signaling (31). C113 does attenuate Hedgehog signaling downstream of smoothened (32), although in vivo studies in mice suggest that it can inhibit a number of tumors via its anti-Wnt effect (33). However, there was significant mortality in animals, given intracardiac injections of pyrvinium, making it an unsuitable treatment (34). Furthermore, it was originally developed for oral administration to target the gastrointestinal tract, and although it is FDA approved, bioavailability and safety are not determined in other contexts. In preliminary studies, C113, which was far less potent than pyrvinium, was better tolerated in vivo, with no toxicities observed. In our previous study using a cutaneous injury of the ear, C113 and XAV-939 showed both reduction in fibrosis and regeneration of auricular (ear) cartilage (14). Nevertheless, XAV-939 showed better potency than C113, making it a good candidate for in vivo OA studies.

We sought to study Wnt signaling and the effect of local therapeutic Wnt inhibition in a murine model of traumatic OA caused by destabilization of the medial meniscus (DMM). To assess the effect of Wnt inhibition on OA progression, we injected the small-molecule Wnt inhibitor, XAV-939, in the intra-articular space. We further studied the cell-specific effects of Wnt modulation in vitro using primary human synovial fibroblasts and chondrocytes in order to understand the cellular and phenotypic basis for the disease-modifying effects.

\section{Results}

Wnt signaling is upregulated in the DMM model of $O A$ and human $O A$ synovial fibroblasts. Previous studies have demonstrated upregulation of Wnt signaling in response to injury in a range of tissue types (35-38). Several studies have linked upregulation of Wnt signaling to cartilage degradation in OA and rheumatoid arthritis (39-43). A frequently used model for traumatic OA is the DMM model in mice that is generated by the transection of the medial meniscotibial ligament, leading to altered stability of the knee joint and subsequent cartilage damage (44). In order to assess whether ablation of Wnt activity can ameliorate the severity of OA in the knee joint, 10-week-old male mice underwent DMM surgery. Three weeks following surgery, the mice were treated with intra-articular injections of either saline control or small-molecule Wnt inhibitor, XAV-939, every 10 days. Knee joints were collected 10 weeks after surgery, 10 days following the last injection (Figure 1A). We assessed the temporal and spatial changes in canonical Wnt/ $\beta$-catenin signaling by performing immunofluorescence of joint sections using an antibody to $\beta$-catenin, a marker of Wnt signaling, and costained with periostin, a stromal/fibroblast marker. Prior to injury, minimal staining was noted; however, after injury, $\beta$-catenin was strongly upregulated in the knee joint, particularly in the synovium (Figure 1B), and was attenuated after XAV-939 treatment (Figure 1C). Our data demonstrated a striking and dynamic increase in canonical Wnt signaling in the synovium, and the enhanced staining was distinctly colocalized with periostin. Isotype controls showed minimal signal (Figure ID). 
A

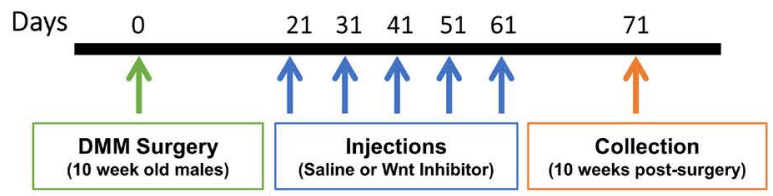

C
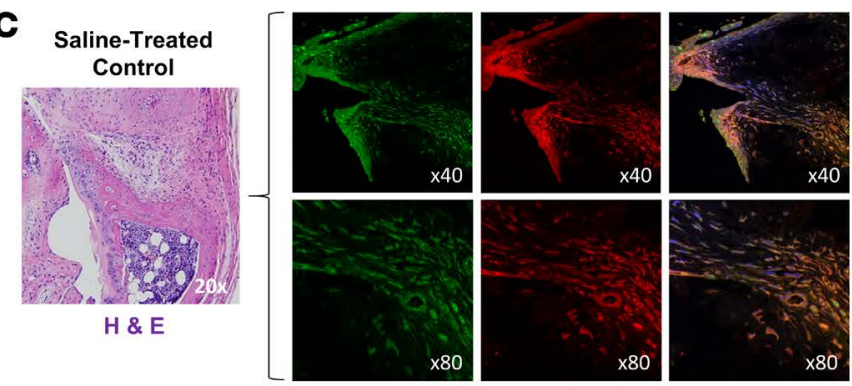

B
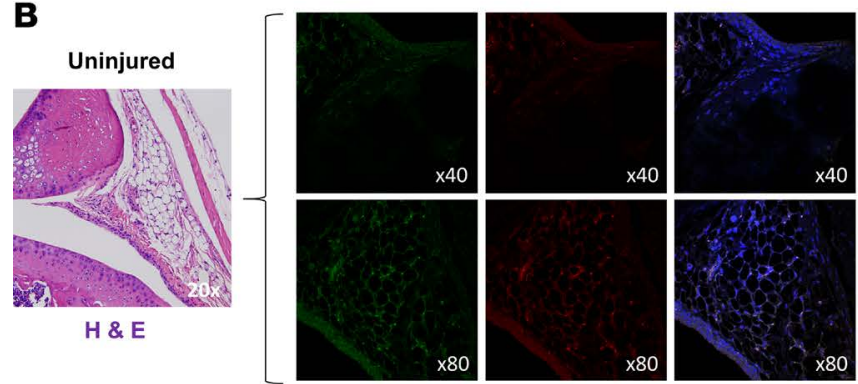

$\times 80$
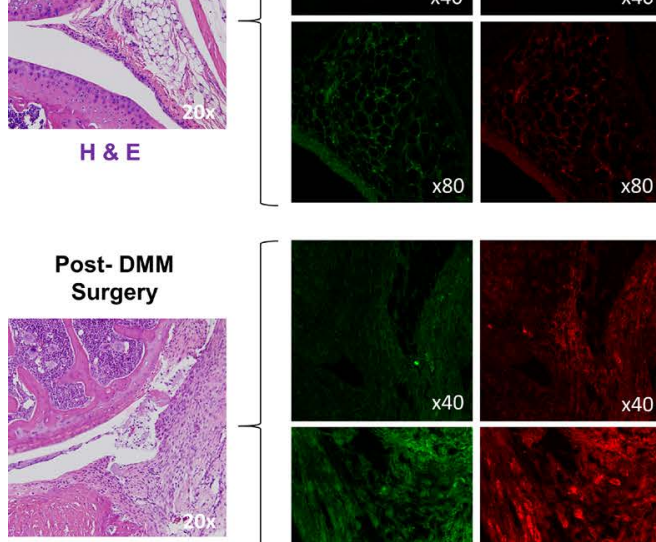

H \& E
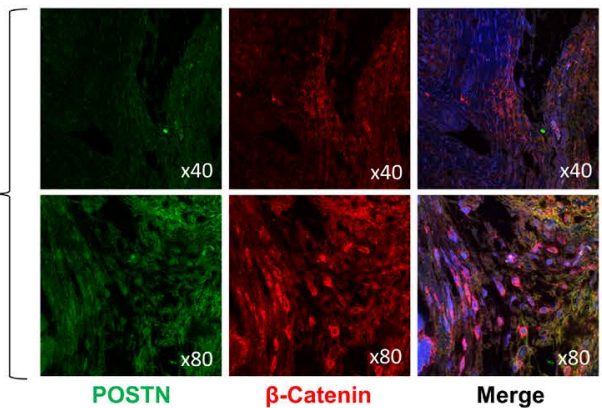
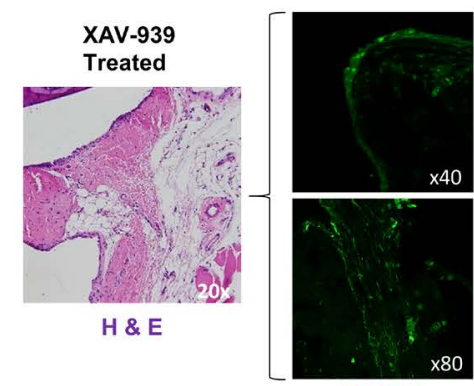

POSTN

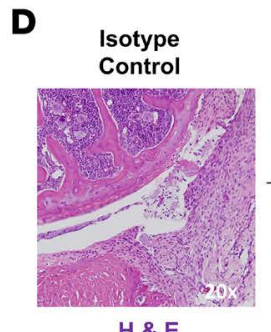

H \& E

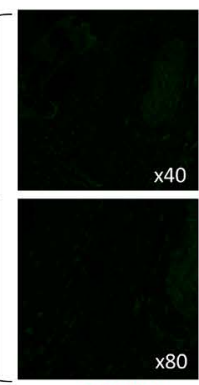

POSTN

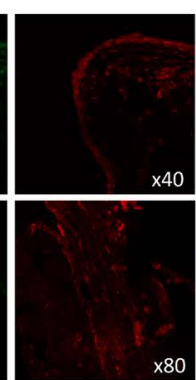

$\beta$-Catenin
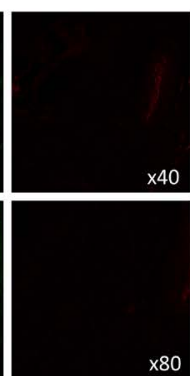

$\beta$-Catenin

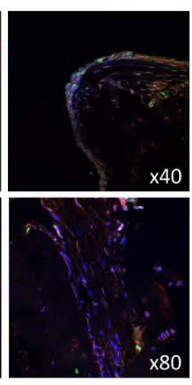

Merge

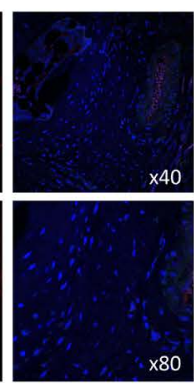

Merge

Figure 1. Wnt signaling is upregulated in the joint after injury. (A) Ten-week-old male mice were subjected to destabilization of the medial meniscus (DMM) surgery. Three weeks after injury, they were injected intra-articularly with saline or Wnt inhibitor every 10 days for a total of 5 times, and knee joints were collected 10 weeks after surgery. (B and C) Synovial tissue was stained by immunofluorescence for $\beta$-catenin (red) and periostin (POSTN) (green), a marker of fibroblasts. DAPI (blue) was used as a counterstain, and yellow indicates costaining of Wnt activation in fibroblasts. (B) Uninjured joints and joints after DMM surgery show upregulation of Wnt/ $\beta$-catenin signaling after injury and increased periostin-positive cells in the synovium. (C) Saline-treated controls, compared with XAV-939-treated joints, indicate that treatment abrogated Wnt/ $\beta$-catenin signaling in synovial fibroblasts. (D) Additional sections from the same joint from after DMM surgery were used as the isotype control. Images from the same joint region were used, although they are from separate sections. The H\&E image is therefore reused from $\mathbf{B}$. The representative isotype control shows no significant staining. Original magnification, $\times 40$ (top rows); $\times 80$ (bottom rows).

In order to determine how long Wnt inhibition using a single dose of inhibitor would persist, NIH3T3 fibroblast cells that overexpress $W n t 3 a$ were treated with both inhibitors. C113 treatment reduced Axin2 expression levels to $6.4 \%$ of their baseline level within 1 hour; XAV-939 treatment reduced Axin2 expression to $3.7 \%$ within 1 hour. Levels of Axin2 for both inhibitors were below $1 \%$ of the starting value after 2 hours and undetectable within 24 hours of treatment. Axin2 was restored to baseline within 72 hours for both inhibitors (Supplemental Figure 1A; supplemental material available online with this article; https://doi.org/10.1172/jci.insight.96308DS1). To determine if intraarticular injections of XAV-939 led to circulating drug, peripheral blood was isolated 3 and 24 hours after intraarticular injection, and XAV-939 was not detectable in plasma, with a detection limit of $0.100 \mathrm{nM}$ (Supplemental Figure 1B). This finding is similar to those in previous studies in which topical administration resulted in nondetectable levels of pyrvinium, C113, or XAV-939 in the plasma (14). Macrophages were very rare in both control and treated joints after injury, indicating an overall minor inflammatory component (Supplemental Figure 2). We did not perform a longitudinal analysis.

To compare basal Wnt signaling as well as capacity for activation in response to Wnt ligands in diseased versus control synovial fibroblasts, we isolated primary human synovial fibroblasts derived from patients with or without OA. As expected, these cells expressed collagen type I, but not collagen type II, 
A

Human Synovial Fibroblasts
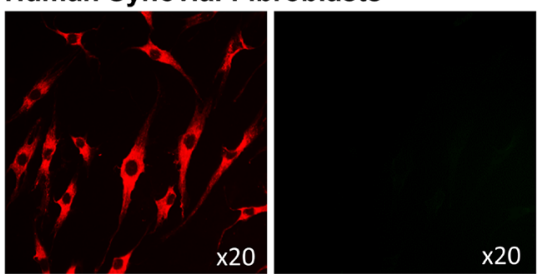

a-COL1A1

a-COL2A1
C

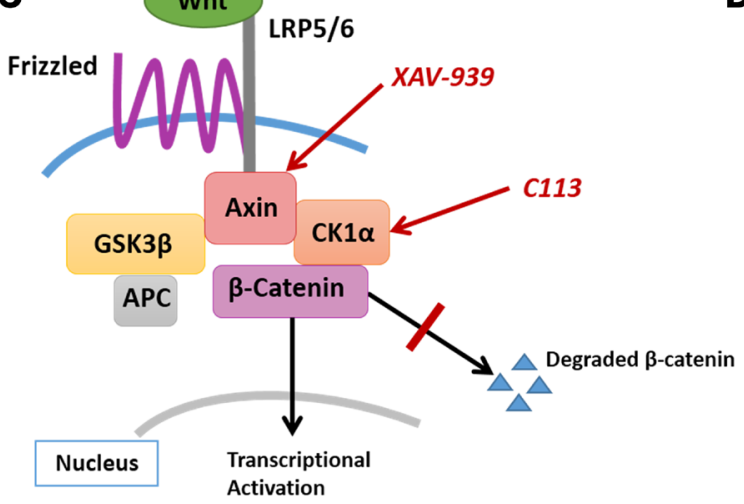

B

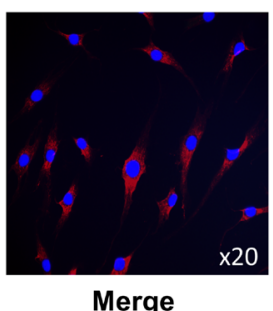

D
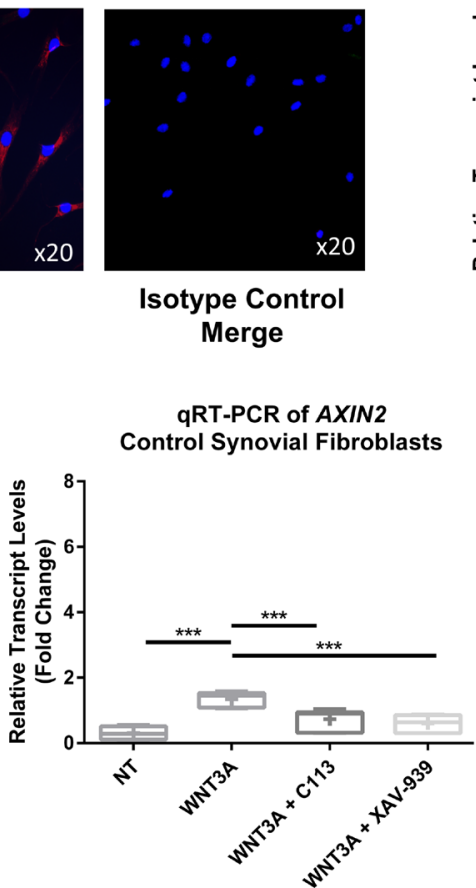

Isotype Control Merge
qRT-PCR of $A X I N 2$

Untreated Synovial Fibroblasts

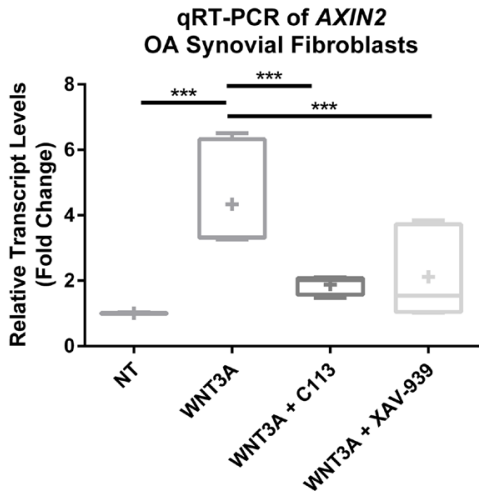

\section{E Human Synovial Fibroblasts}
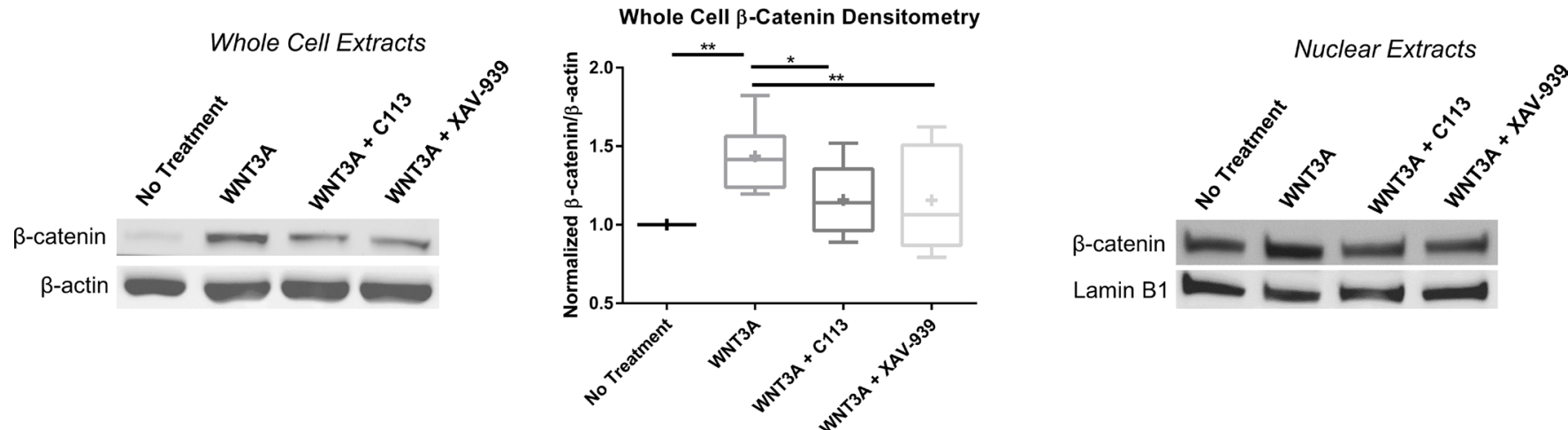

Figure 2. Synovial fibroblasts are responsive to Wnt inhibition treatment. (A) Synovial fibroblasts isolated from patients with osteoarthritis (OA) were positive for expression of type I collagen (red) but not type II collagen (green). Nuclei were stained with DAPI (blue). Isotype controls were negative. Original magnification, $\times 20$. (B) OA synovial fibroblasts were compared with control synovial fibroblasts by real-time qRT-PCR of AXIN2, a downstream marker of Wnt signaling. Expression was higher in synovial fibroblasts isolated from patients with OA than those from controls. Statistical analysis was performed using a $t$ test, ${ }^{* * *} P<0.001, n=6$. (C) Schematic of the Wnt/ $\beta$-catenin pathway showing target molecules inhibited by the Wnt inhibitors (C113 and XAV-939) used in the study. (D) Furthermore, synovial fibroblasts were treated with recombinant WNT3A with and without Wnt inhibitors (C113 and XAV-939) and analyzed by real-time RT-PCR of AXIN2. Wnt signaling was ameliorated by both inhibitors in control and OA synovial fibroblasts. One-way ANOVA with Tukey's post-hoc test was used to compare treatment groups, ${ }^{* * *} P<0.001, n=6$. (E) Immunoblotting of $\beta$-catenin in $0 A$ synovial fibroblast cell lysates after treatment with recombinant WNT3A with or without inhibitors. Densitometry of immunoblots was performed to quantify the reduction of Wnt signaling after inhibitor treatment. One-way ANOVA with Tukey's post-hoc test was used to compare treatment groups, ${ }^{*} P<0.05,{ }^{* *} P<0.01, n=$ 8. Alteration of $\beta$-catenin signaling in the nucleus was confirmed by immunoblotting of synovial fibroblast nuclear lysates compared with lamin B1 loading control. (B, D, and E) For data presented as box-and-whiskers plots, horizontal lines indicate the medians, cross marks indicate the means, boxes indicate the 25 th to 75 th percentiles, and whiskers indicate the minimum and maximum values of the data set.

as shown by immunofluorescent staining (Figure 2A). Quantitative real-time RT-PCR was performed on cDNA collected from these fibroblasts to assess levels of AXIN2 transcripts, a direct target of the Wnt signaling pathway that is often used as a marker to assess the intensity and duration of canonical Wnt activation (45) (Figure 2B). Synovial fibroblasts isolated from OA patients had higher basal levels of Wnt activity. These cells were then stimulated with WNT3A recombinant protein to stimulate Wnt pathway activity in the absence or presence of two small-molecule Wnt inhibitors, C113 and XAV-939, 
which inhibit Wnt activity through two distinct mechanisms $(30,46)$ (Figure 2C). In both control and OA cells, Wnt pathway activation was significantly attenuated with both inhibitors (Figure 2D). We further confirmed these data by analyzing levels of $\beta$-catenin in the total cell lysates and nuclear fraction by immunoblotting (Figure 2E).

To assess the contribution of noncanonical Wnt signaling, we stained murine synovium, human synovial fibroblasts, murine cartilage, and human chondrocytes with an antibody to active Jnk by immunofluorescence. Relatively low levels of staining were observed, with no discernible changes between treatment groups in murine tissue or human cells (Supplemental Figure 3).

Inhibition of Wnt signaling in vivo ameliorates $O A$ severity. To visualize the severity of cartilage damage in the injured knees, Safranin O/Fast Green staining was utilized (Figure 3A), and quantification of articular cartilage damage was determined using Osteoarthritis Research Society International (OARSI) scoring (47) by two blinded observers (Figure 3B). Results showed marked protection in the severity of cartilage degeneration and significant reduction in the OARSI scores in both medial tibial plateau and medial femoral condyle in the Wnt inhibitor-treated group compared with saline-treated controls. Chondrocyte cellularity analysis further showed greater chondrocyte loss in the saline-treated group compared with the Wnt inhibitor-treated group (Figure 3C). To assess synovitis, Masson's trichrome staining was performed, and the degree of synovitis was blindly scored as described previously (48) (Figure 3, D and E). Results showed a marked reduction in the degree of synovitis in the Wnt inhibitor-treated group compared with the saline-treated group. We also performed subchondral bone plate histomorphometry in the Wnt inhibitor-treated group compared with the saline-treated group as previously described (49). Results showed no significant differences in the subchondral bone plate thickness in the Wnt inhibitor-treated group compared with the saline-treated group (Supplemental Figure 4).

Wnt inhibition reduces both proliferation and type I collagen levels of synovial fibroblasts. We sought to assess the cellular effects of Wnt inhibitors on synovial fibroblasts to clarify the basis for the observed decrease in synovitis. Increased proliferation of synovial fibroblasts has been shown in the context of OA and rheumatoid arthritis (50-52). We identified that treatment with XAV-939 reduced the numbers of proliferating fibroblasts in the synovium, as assessed by immunostaining with Ki-67 and PCNA on sections from control- or XAV-939-treated mice after DMM injury (Figure 4, A and B). To determine if Wnt directly modulated synovial fibroblast proliferation, we assessed the proliferation response of isolated human synovial fibroblasts by BrdU incorporation assay (Figure 4C). Synovial fibroblasts from OA patients showed significantly higher basal levels of proliferation than control fibroblasts from healthy controls. While both control and OA synovial fibroblasts demonstrated an increase in proliferation with the addition of WNT3A, the effect was only significant in control synovial fibroblasts. The elevated basal proliferation rate of OA synovial fibroblasts may be an explanation for the lack of response in the presence of WNT3A. Importantly, both small-molecule Wnt inhibitors resulted in a statistically significant decrease in proliferation of OA synovial fibroblasts to below basal levels. The antiproliferative effect of XAV-939 and C113 on control synovial fibroblasts demonstrated a decreased trend but did not achieve statistical significance.

The primary constituent of synovial fibrotic matrix is collagen type I, which is produced by synovial fibroblasts (53). Since there was a striking effect of XAV-939 treatment on synovitis, we further assessed the effect of Wnt modulation on type I collagen synthesis of synovial fibroblasts in vitro. Treatment of cultured synovial fibroblasts with WNT3A ligand resulted in a significant increase in COL1A1 transcript levels, and this increase was not only abrogated with the addition of either Wnt inhibitor, but levels were reduced to below baseline $(\mathrm{C} 113=43.77 \%$, XAV-939 $=83.09 \%)$ (Figure $5 \mathrm{~A})$. As further confirmation, we demonstrated the same trends in collagen type I protein expression (Figure 5B). Taken together, Wnt inhibitors addressed two key aspects of synovial fibrosis by reducing both proliferation of synovial fibroblasts and their synthesis of type I collagen.

Effect of Wnt modulation on OA-derived human articular chondrocytes. We sought to evaluate Wnt-mediated effects on cartilage/chondrocytes. To determine whether XAV-939 treatment was inducing proliferation of chondrocytes in the articular cartilage, we performed immunostaining of both Ki-67 and PCNA on control or treated murine joints (Figure 6, A-C). Staining of the joints showed no significant difference in the proliferative activity of the chondrocytes. To further confirm that Wnt inhibition did not induce proliferation, chondrocytes were isolated from the articular cartilage of patients with OA and were verified by expression of the chondrocyte-specific marker, collagen type II (Figure 6D). We utilized a BrdU incorporation assay 
A
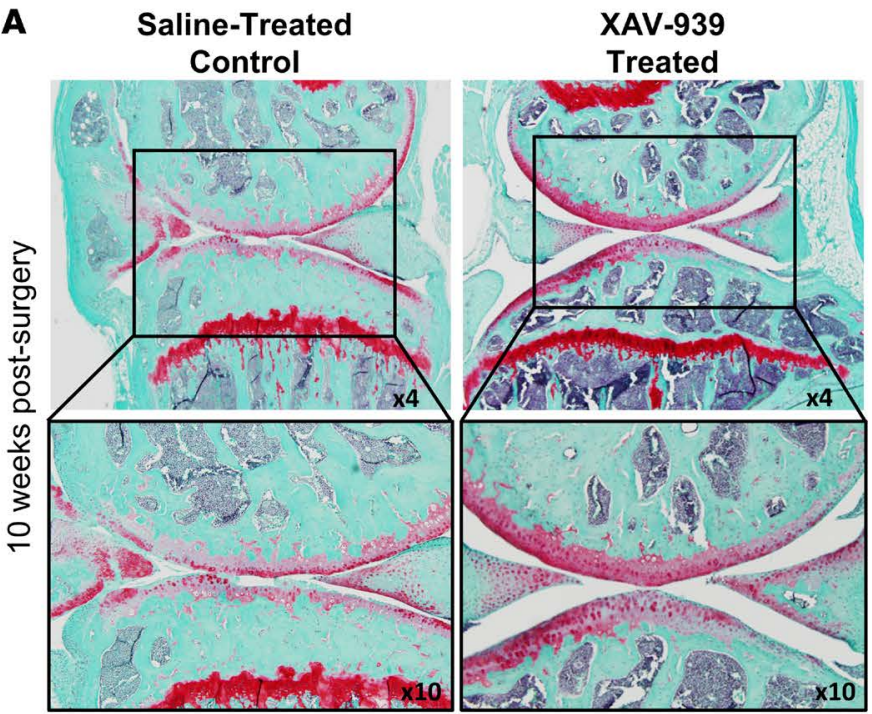

D
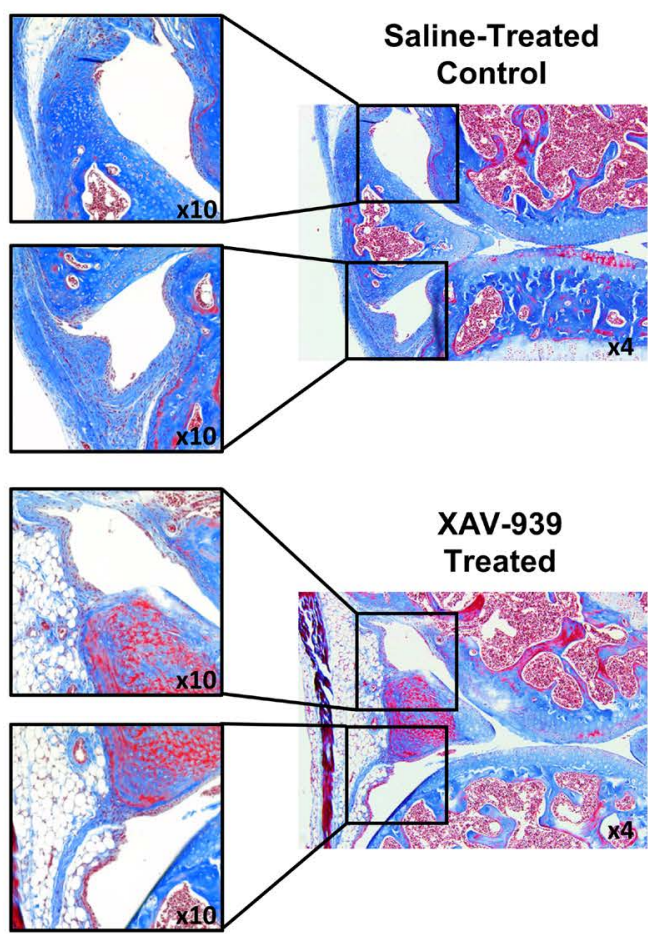

B

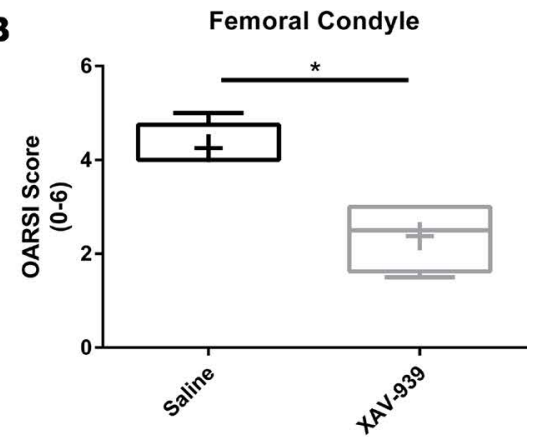

Tibial Plateau

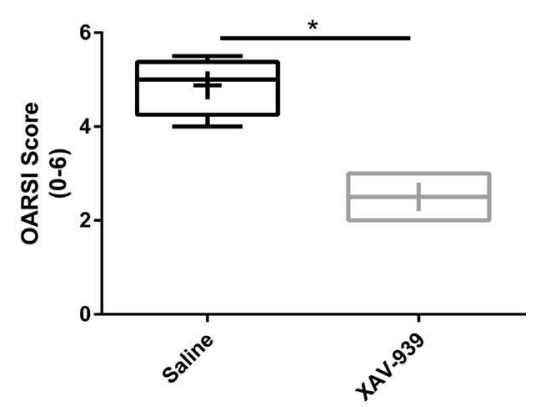

C

\section{Chondrocyte Cellularity}

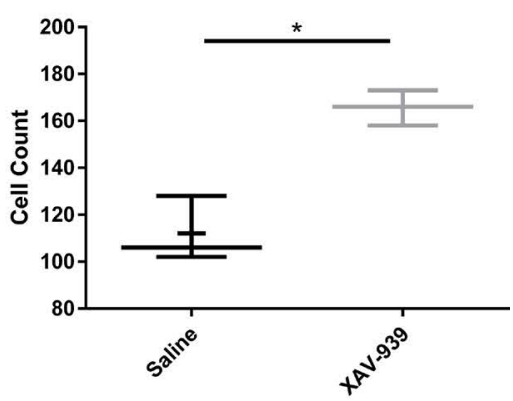

E

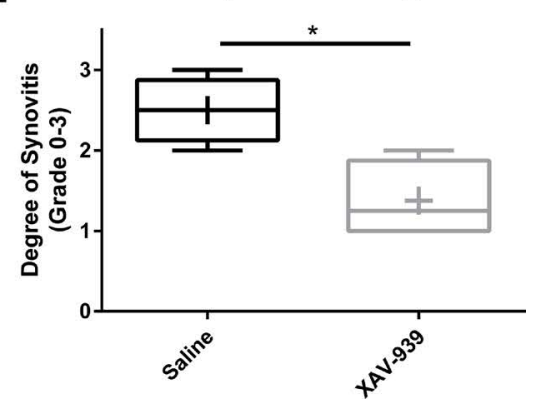

Figure 3. Wnt inhibition reduced cartilage degeneration and synovitis in DMM model. (A) Following destabilization of the medial meniscus (DMM) surgery and Wnt inhibitor or saline control treatment, Safranin O/Fast Green staining performed on knee joints showed a reduction in the degree of cartilage degeneration with treatment. Original magnification, $\times 4$ (top row); $\times 10$ (bottom row). (B) The medial femoral condyle and medial tibial plateau were scored using the Osteoarthritis Research Society International (OARSI) system by two independent blinded readers, and both regions had significantly lower scores with Wnt inhibition compared with saline group. (C) Chondrocyte cellularity was significantly reduced in saline-treated group compared with Wnt inhibitor-treated group. ( $\mathbf{D}$ and $\mathbf{E}$ ) Masson's trichrome staining further showed significant reduction in the degree of synovitis in Wnt inhibitor-treated group compared with saline-treated group. Original magnification, $\times 10$ (left); $\times 4$ (right). (B, C, and E) Statistical analysis was performed using a $t$ test, ${ }^{*} P<0.05, n=8$. For data presented as box-and-whiskers plots, horizontal lines indicate the medians, cross marks indicate the means, boxes indicate the 25 th to 75 th percentiles, and whiskers indicate the minimum and maximum values of the data set. 
and noted no significant differences in the proliferative activity of the cells with Wnt pathway activation or inhibition, supporting our finding that the cartilage-sparing effects observed with XAV-939 treatment were not due to promoting chondrocyte regeneration (Figure $6 \mathrm{E}$ ).

We next determined whether chondrocytes were responsive to Wnt signaling by evaluating AXIN2 transcript levels by real-time RT-PCR (Figure 6F). AXIN2 expression was activated $>22$-fold 24 hours after activation with WNT3A, and addition of either XAV-939 or C113 significantly reduced Wnt signaling activation, as gauged by AXIN2 transcript levels. We next assessed if the cartilage-sparing effects of Wnt inhibitors may be modulating expression of type II collagen matrix or the expression of lubricin, encoded by $P R G 4$, a proteoglycan secreted by chondrocytes that has been shown to play a positive role in OA in animal models (54-57). Our data showed a significant decrease in COL2A1 transcript levels after the addition of WNT3A, while Wnt inhibition rescued the mRNA expression (Figure 6G). Transcript levels of PRG4 were also significantly increased by either C113 or XAV-939 treatment, suggesting a positive effect on expression (C113 = 11.693-fold increase, XAV-939 $=2.448$-fold increase) (Figure 6H). Collectively, the cartilage-sparing effects of XAV-939 and C113 were not likely due to spurring chondrocyte/cartilage regeneration. However, enhancing type II collagen matrix and specific proteoglycan secretion may contribute to the disease-ameliorating phenotype.

We further sought to determine if the secretome of synovial fibroblasts had apparent effects on the chondrocyte phenotype. Human OA synovial fibroblasts were treated with WNT3A, WNT3A plus C113, or WNT3A plus XAV-939. Conditioned media were collected 24 hours following treatment and then transferred to human chondrocytes. No changes were observed in apoptosis, hypertrophy (indicated by qRT-PCR of $C O L X)$, or proteinase levels (indicated by qRT-PCR of MMP13). Additionally, conditioned media were analyzed by cytokine array, with few notable significant changes between treatment groups, indicating minimal significant secretome modulation by Wnt signaling in synovial fibroblasts (Supplemental Figure 5 and Supplemental Table 1).

\section{Discussion}

Our study demonstrated that traumatic joint injury through DMM surgery induced robust activation of canonical Wnt signaling, most striking in the synovium, and this upregulation was downregulated with intermittent (every 10 days), local (intra-articular) treatment using a small-molecule Wnt inhibitor, resulting in amelioration of both synovitis and cartilage loss. An important advantage of identifying therapeutic benefits with local administration is avoiding systemic effects of Wnt inhibition on Wnt-dependent tissues, such as intestinal stem cell/intestinal turnover, hematopoiesis, and bone density (58). The use of small-molecule Wnt inhibitors bypasses the restrictions of genetic approaches by targeting the entire injury milieu, rather than a particular cell type, as is the case in genetic models (26). Additionally, since different cell types exhibit different levels of Wnt activation and sensitivity to Wnt inhibition, one can also fine-tune the degree and timing of Wnt inhibition using dosing strategies that are calibrated. This is exemplified in the cutaneous wound model, in which the dose of the inhibitor utilized primarily reduced Wnt signaling in the dermis without significant reduction in the epidermis (14). Our findings indicated that there was continued Wnt signaling elevation 10 weeks after DMM surgery within the synovium, suggesting that Wnt activation following injury did not return to basal levels after the initial damage response. This was further confirmed by our analysis of human OA patient-derived synovial fibroblasts, which demonstrated elevated basal Wnt signaling levels as compared with control synovial fibroblasts, suggesting that Wnt signaling is chronically elevated in the synovial cells of OA patients. There have not been reports identifying Wnt effectors activated and sustained following injury, although specific Wnt effectors, such as Wnt16, a weak canonical activator, have been shown to increase following injury but return completely to basal levels in DMM-operated knees after 8 weeks (59). Sustained Wnt activation following injury, as observed in the synovium after DMM injury, is in contrast to cutaneous injury and ischemic myocardial injury models in which the activation was transient $(14,15)$ and may reflect the progressive nature of the disease. Although published studies using mice suggest that Wnt target gene expression is elevated after DMM in chondrocytes/cartilage $(23,59)$, we did not observe a notable upregulation of Wnt signaling in the cartilage by immunofluorescent staining for $\beta$-catenin, likely due to reduced sensitivity of immunofluorescence. These data highlight that changes in Wnt signaling following DMM were more dynamic and robust in the synovium as compared with cartilage. 
A

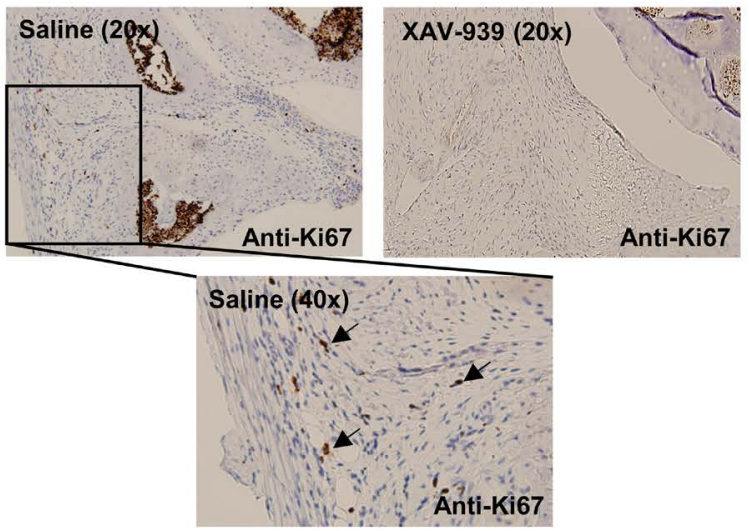

C Synovial Fibroblast Proliferation

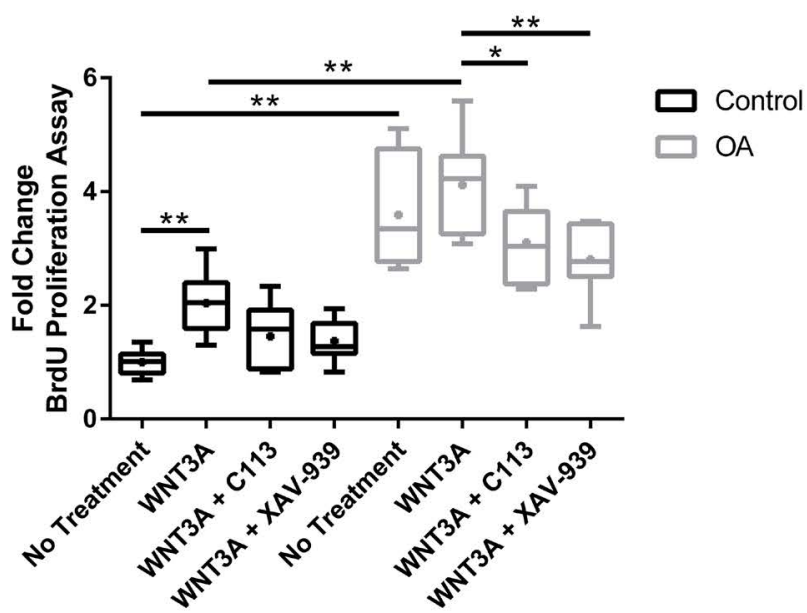

B
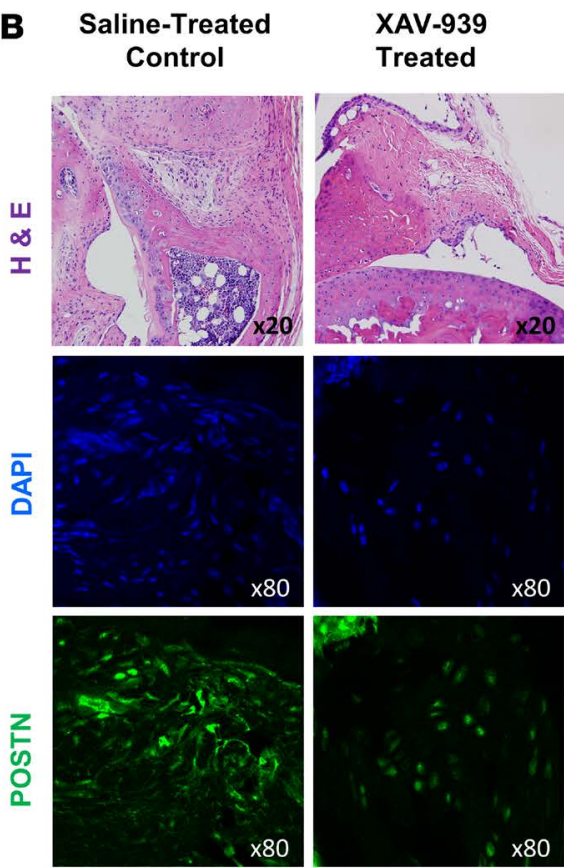

XAV-939

Treated
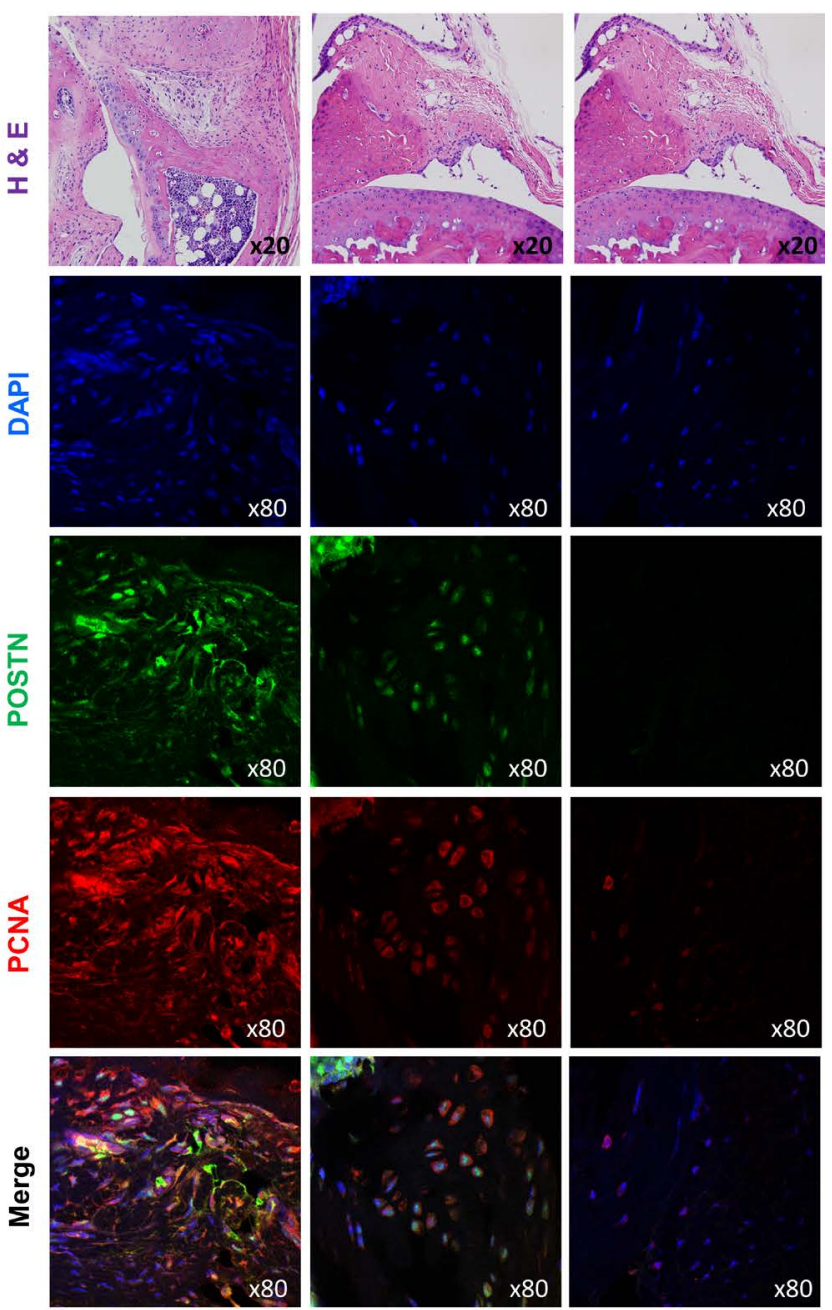

Figure 4. Proliferation of synovial fibroblasts is elevated in $\mathrm{OA}$ and can be moderated through Wnt inhibition. (A) Immunohistochemistry of Ki-67 on knee synovia from saline control and Wnt inhibitor mice 10 weeks after surgery showed a reduction in proliferation of synovial fibroblasts after treatment with Wnt inhibitor. Original magnification, $\times 20$ (top); $\times 40$ (bottom). (B) Knee synovia were stained by immunofluorescence with periostin (P0STN, green) and PCNA (red), a proliferating cell marker, and counterstained with nuclear DAPI (blue). The joint region used for immunofluorescence is shown by H\&E stain. Synovia from saline-treated control mice demonstrated increased levels of periostin- and PCNA-positive cells, when compared with XAV939-treated synovia. Additional sections from the same joint from the XAV-939-treated mouse were used as the isotype control. Images from the same joint region were used, although they are from separate sections. The H\&E image is therefore reused. The representative isotype control shows minimal staining. Original magnification, $\times 20$ (top row); $\times 80$ (bottom 4 rows). (C) Proliferation was assessed by BrdU assay on synovial fibroblasts. Basal proliferation levels were significantly upregulated in OA patient fibroblasts compared with controls. Furthermore, WNT3A treatment of control fibroblasts also significantly upregulated proliferation. Wnt inhibition in OA fibroblasts decreased the proliferation rate. Two-way ANOVA with Tukey's post-hoc test was performed, ${ }^{*} P<0.05,{ }^{*} P<0.01, n=6$. For data presented as box-and-whiskers plots, horizontal lines indicate the medians, cross marks indicate the means, boxes indicate the 25 th to 75 th percentiles, and whiskers indicate the minimum and maximum values of the data set.

Extensive investigation of the Wnt/ $\beta$-catenin pathway using transgenic mouse models suggests that Wnt/ $\beta$-catenin signaling needs to be carefully modulated for cartilage development as well as maintenance of normal articular cartilage function $(21,60)$. Both excessive Wnt signaling and Wnt suppression lead to chondrocyte pathologies and cartilage loss in mice as well as humans $(19,21,61-63)$. Studies that have examined activation of Wnt signaling in chondrocytes and synovium in vivo have noted stimulation of MMP3 and MMP13 and a catabolic profile in chondrocytes, though the precise genes altered differ based on the Wnt ligand and/or receptor $(18,19,63,64)$. Overexpression of $D k k 1$, a negative regulator of the Wnt signaling pathway, in cartilage via the Col2a1 promoter protected against cartilage destruction. Furthermore, an ongoing phase II clinical trial of SM05690 (Samumed), a small-molecule 
A

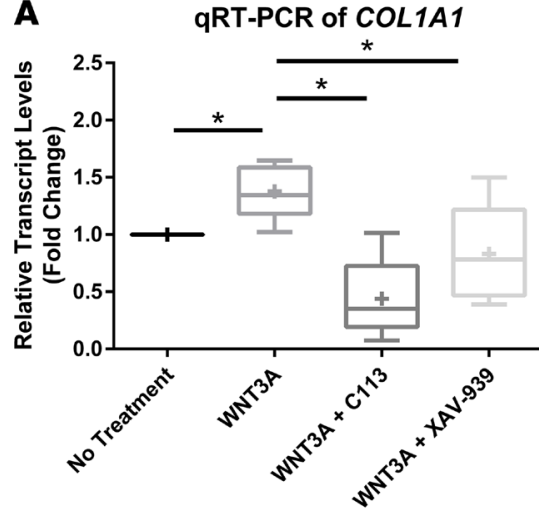

B Human Synovial Fibroblasts

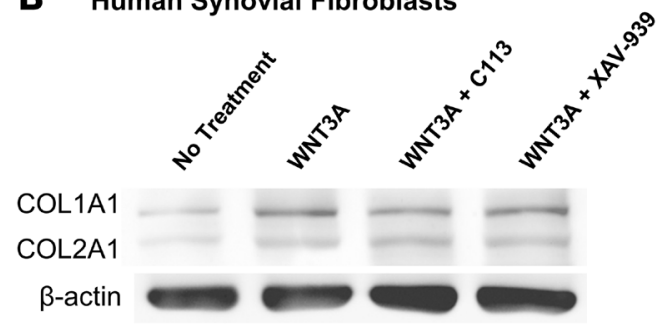

COL1A1 Densitometry

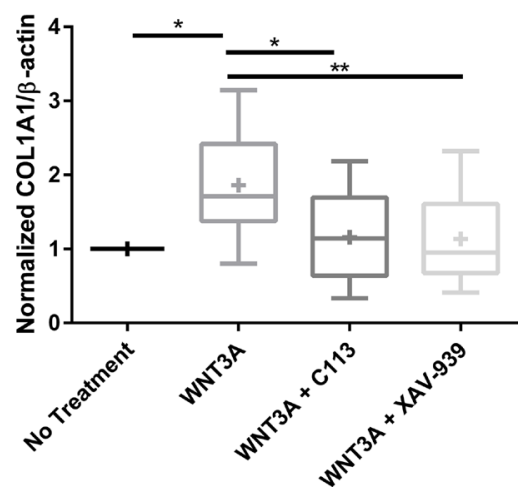

Figure 5. Wnt inhibitor treatment results in decreased collagen type I expression. (A) Real-time qRT-PCR of COL1A1 on synovial fibroblasts isolated from osteoarthritis (OA) patients shows an increase after recombinant WNT3A treatment and a decrease after Wnt inhibitor treatment. One-way ANOVA with Tukey's post-hoc test was performed, ${ }^{*} P<0.05, n=5$. (B) Immunoblotting of type I collagen in OA synovial fibroblasts also demonstrates an increase after activation of Wnt signaling and a decrease in response to $\mathrm{C113}$ or XAV-939 inhibitors. Densitometry was used to quantify COL1A1 protein levels normalized to the $\beta$-actin loading control. One-way ANOVA with Tukey's post-hoc test was performed, ${ }^{*} P<0.05,{ }^{* *} P<0.01, n=8$. For data presented as box-and-whiskers plots, horizontal lines indicate the medians, cross marks indicate the means, boxes indicate the 25th to 75 th percentiles, and whiskers indicate the minimum and maximum values of the data set.

inhibitor of Wnt signaling, is applied intra-articularly in moderate-to-severe knee OA (identification no. NCT03122860). Preclinical studies reported improved outcome through both reduced cartilage degeneration and reduction in inflammatory cytokine and protease production, and no toxicity was noted (65). Our cartilage-sparing effects in vivo using XAV-939 and our in vitro data from isolated human chondrocytes exposed to XAV-939 and C113 were consistent with these genetic studies in that we observed that Wnt inhibitors acted to restore a chondrocyte-protective profile and reverse the catabolic profile following Wnt activation. For example, lubricin (PRG4) and type II collagen are essential for joint homeostasis, and their disruption results in cartilage breakdown (66-68). Expression of PRG4 and COL2A1 increased with Wnt inhibition, supporting the prior studies linking Wnt activation to a catabolic phenotype in chondrocytes, which can be reversed with Wnt inhibition. PRG4 expression has been shown to be protective in OA and, furthermore, has been shown to upregulate hypoxia-inducible factor $3 \alpha$, which inhibits transcriptional programs that promote cartilage catabolism and hypertrophy $(55,69)$. We did not observe changes in $M M P 13$, as previously reported with Wnt modulation of chondrocytes, and this may be due to dosing and other differences in the experimental context. Such differences may also be attributed to sustained versus acute inhibition of this pathway. While Wnt inhibition significantly reduced the proliferation of OA synovial fibroblasts, it did not appear to alter proliferation of OA chondrocytes. Our study suggests that Wnt inhibition spared cartilage loss through mechanisms other than increased chondrocyte proliferation and true regeneration.

Excessive synovial fibroblast proliferation and deposition of type I collagen are hallmarks of synovitis (70). Herein, intra-articular injection of XAV-939 was shown to result in significantly reduced synovitis. Concurrently, type I collagen deposition was vastly reduced. Consistent with these findings, primary human synovial fibroblasts demonstrated heightened proliferation and type I collagen synthesis in response to WNT3A. Both proliferation and collagen synthesis were significantly reduced with both XAV-939 and $\mathrm{C} 113$, suggesting that these are direct targets of Wnt signaling. To our knowledge, this is the first report to identify synovial fibroblasts as key targets of Wnt modulation and to identify their proliferation and matrix synthesis as direct Wnt-modulated cellular effects. Although earlier studies in which $D k k 1$ was overexpressed under the Colla1 promoter (which would target expression in matrix-synthesizing synoviocytes) demonstrated reduced cartilage lesions and osteophytes as well as reduced overall OA score, specific evaluation of the effect of Wnt inhibition in cellular phenotype of synovial fibroblasts was not reported (24). Our findings in vivo were further supported using mechanistically distinct Wnt inhibitors to assess the cellular responsiveness to Wnt inhibition on human synovial fibroblasts in culture. We demonstrated that basal Wnt signaling as well as the basal proliferation rate was higher in synovial fibroblasts derived from OA patients. This elevation in Wnt signaling may be responsible for the higher baseline proliferation of 
A
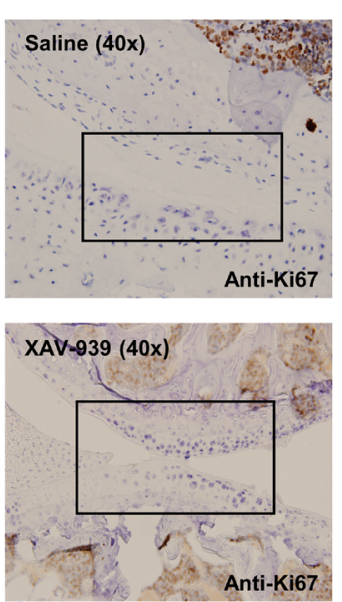

B

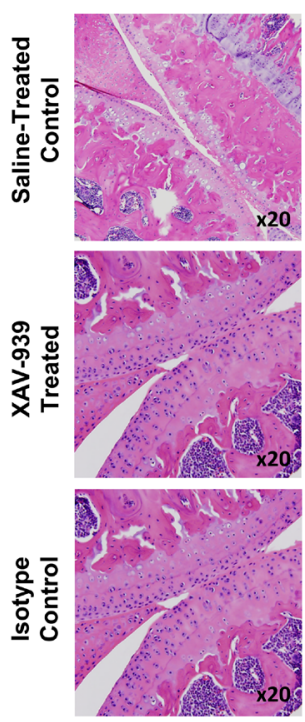

$H$ \& $E$
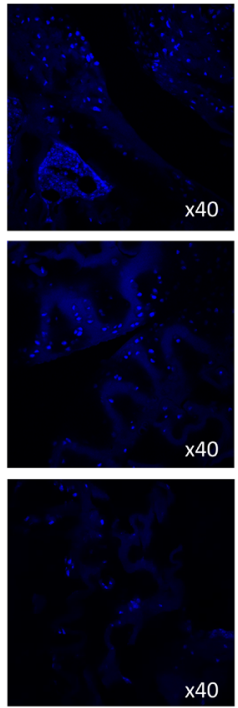

DAPI
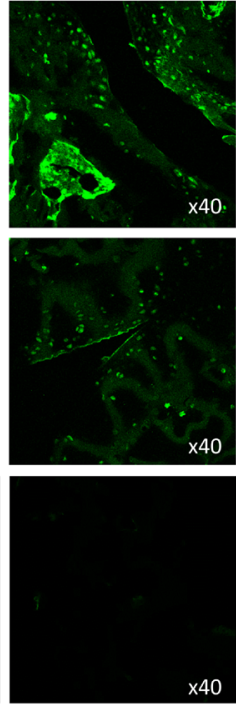

sox9
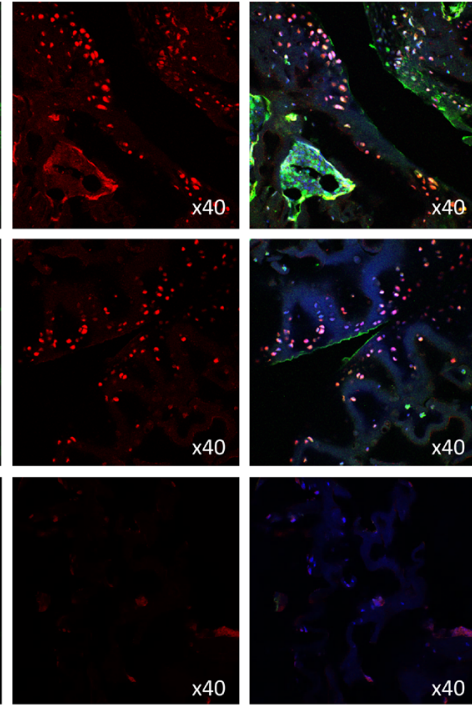

PCNA

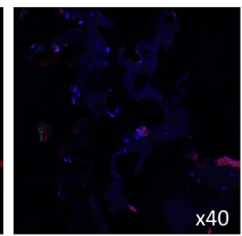

Merge
C

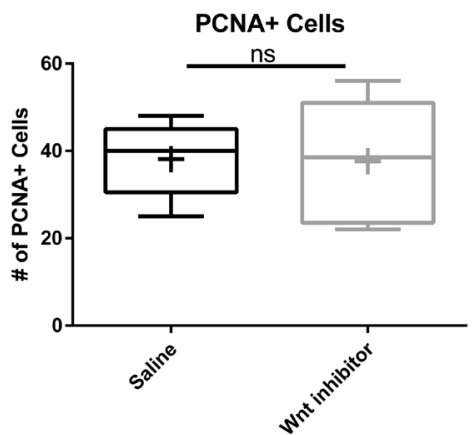

D

Human Chondrocytes

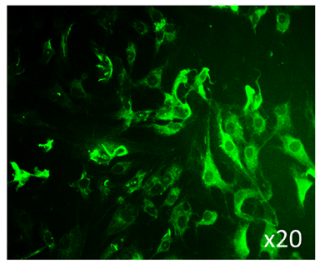

a-COL2A1

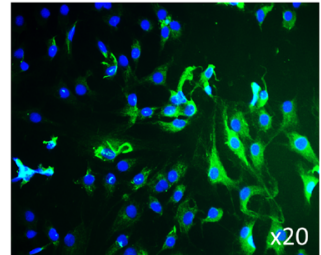

$\mathrm{DAPI}+\alpha-\mathrm{COL} 2 \mathrm{~A} 1$

DAPI $+\alpha-C O L 2 A 1$ Isotype Control

G
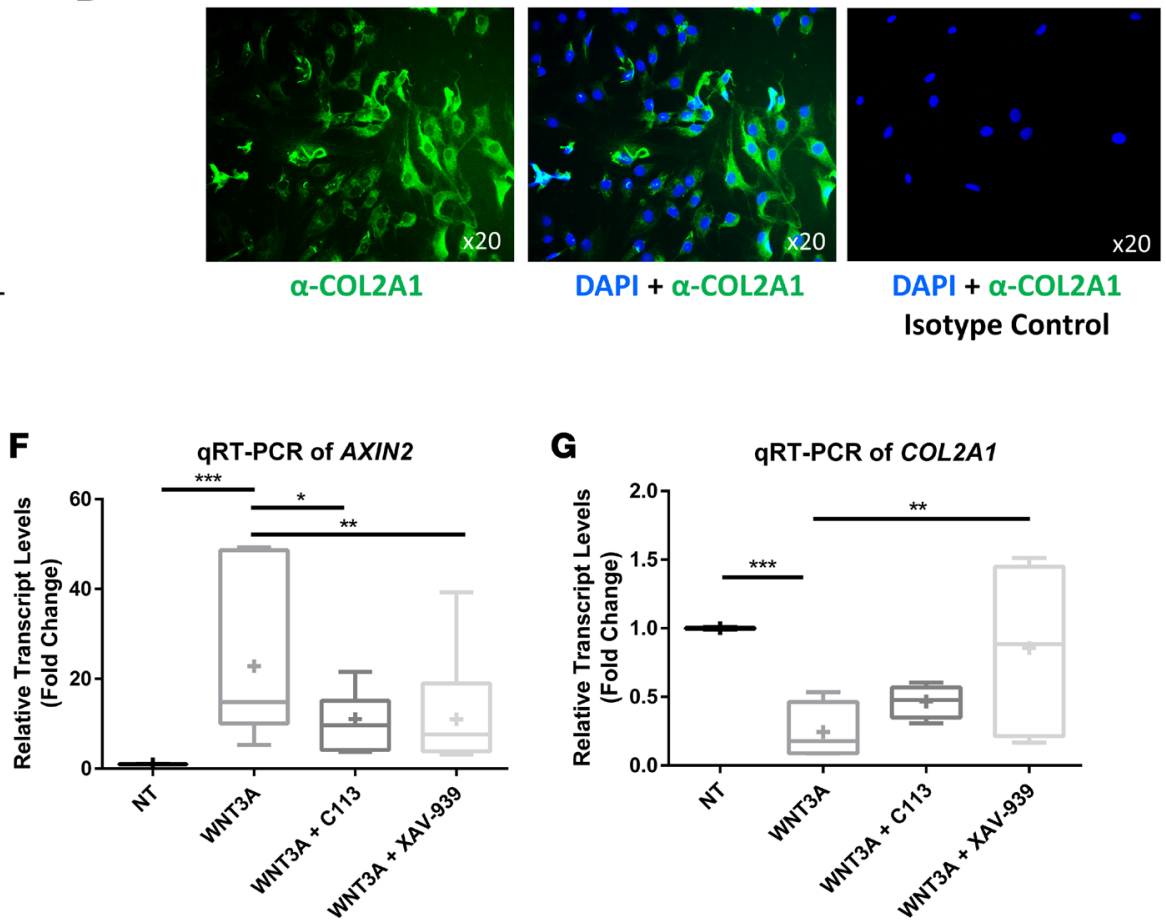

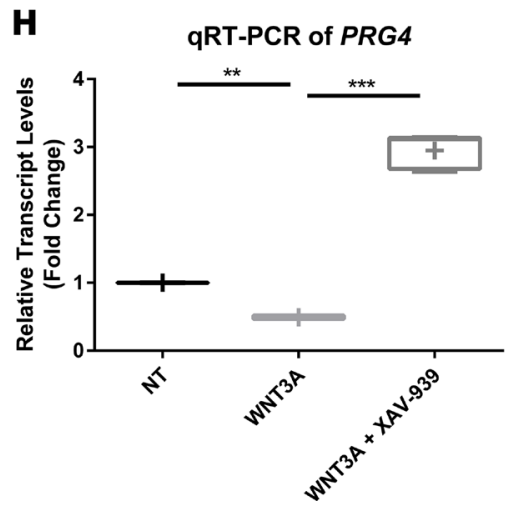

E

Chondrocyte Proliferation

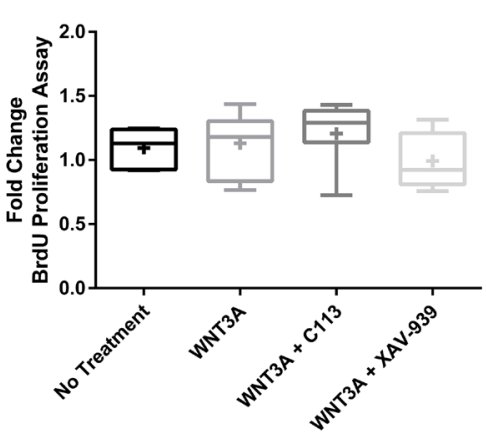

qRT-PCR of PRG4

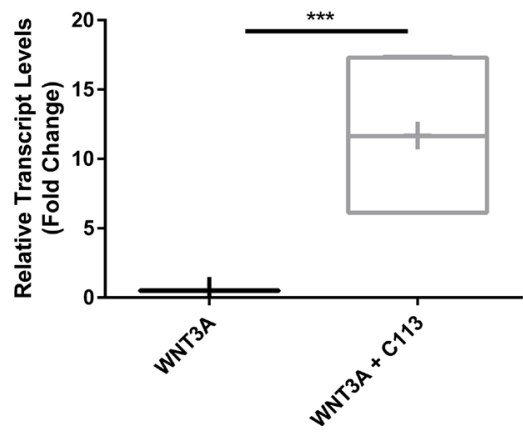


Figure 6. Wnt inhibitor treatment of chondrocytes does not alter proliferation but modifies gene expression. (A) Immunohistochemistry of Ki-67 in the joint from control and Wnt inhibitor mice 10 weeks after surgery demonstrated no detectable proliferation of chondrocytes after treatment with Wnt inhibitor. Original magnification, $\times 40$. (B) Joints were stained with H\&E and also by immunofluorescence with SOX9 (green), a marker for chondrocytes, and PCNA (red), a proliferating cell marker, and counterstained with DAPI (blue). Additional sections from the same joint from the XAV-939-treated sample were used as the isotype control. Images from the same joint region were used, although they are from separate sections. The H\&E image is therefore reused. Isotype controls showed background but no nuclear staining. Original magnification, $\times 20$ (first column); $\times 40$ (rightmost 4 rows). (C) Costained PCNA-positive and SOX9-positive cells were quantified, and no significant difference was detected between control and treated groups. Statistical analysis by $t$ test was performed, $P>0.05, n=3$. (D) Chondrocytes were isolated from patients with osteoarthritis (OA) and stained by immunofluorescence for type II collagen (green) and DAPI (blue) to confirm phenotype. Isotype controls did not show any collagen type II staining. Original magnification, $\times 20$. (E) Proliferation was assessed by BrdU assay and showed no response in proliferation with Wnt treatment or inhibition. (F) Real-time qRT-PCR of AXIN2 demonstrates that chondrocytes are responsive to Wnt activation and inhibition by C113 and XAV-939. (G) Real-time qRT-PCR of COL2A1 demonstrates that type II collagen transcripts are downregulated in response to Wnt expression and increased after inhibition. (H) Real-time qRT-PCR of PRG4 demonstrates that Wnt inhibition in chondrocytes increases PRG4 transcript levels. (E-H) Statistical analysis was performed using a 1-way ANOVA with Tukey's post-hoc test, ${ }^{*} P<0.05,{ }^{*} P<0.01,{ }^{* *} P<0.001, n=5$. For data presented as box-and-whiskers plots, horizontal lines indicate the medians, cross marks indicate the means, boxes indicate the 25 th to 75 th percentiles, and whiskers indicate the minimum and maximum values of the data set.

OA-derived synovial fibroblasts, as their proliferation rate was only minimally responsive to WNT3A, in contrast to control synovial fibroblasts. Both XAV-939 and C113 mediated decreased proliferation and decreased expression of type I collagen. Interestingly, despite synovial hyperplasia in the DMM model of OA, there was minimal evidence of both myofibroblasts and cellular inflammation. We also did not observe statistically significant Wnt-dependent modulation of MMP13 or MMP1 in human synovial fibroblasts (data not shown). We observed that WNT3A increased MMP13 transcripts slightly ( 1.3-fold, albeit statistically significantly, $P<0.05)$ in human chondrocytes; however, we did not observe a statistically significant abrogation of the increased transcript levels with XAV-939 or C113 (data not shown). Hence, we cannot extrapolate the effect of therapeutic Wnt inhibition on disease severity on OA models that exhibit a significant inflammatory component.

Our data support a role for therapeutic Wnt inhibition in the treatment of traumatic OA and suggest that the disease-mitigating effects of Wnt inhibition may be due to effects on both synovial fibroblasts and chondrocytes, although it is difficult to discern if the cartilage-sparing effects are primarily due to direct effects on articular cartilage or secondary to effects on synovial tissues. Our findings particularly underscore the antifibrotic effects of therapeutic Wnt inhibition following injury observed in other models of therapeutic Wnt downregulation (13-15, 34).

\section{Methods}

Animal model. All mice were obtained from The Jackson Laboratory. Ten-week-old male C57BL/6J mice were subjected to DMM surgery to induce OA as described previously $(71,72)$. Three weeks after surgery, five intra-articular injections of XAV-939 $(0.4 \mathrm{mM}$, Seleck Chemicals, S1180) or saline were administered at 10-day intervals, with a total volume of $5 \mu$. Knee joints from control $(n=8)$ and Wnt inhibitor mice $(n=8)$ were collected at 10 weeks after surgery.

Histology. Joints were fixed overnight in TissuFix (Chaptec), decalcified for 1.5 hours in RDO Rapid Decalcifier (Apex Engineering), and further fixed in TissuFix overnight, followed by embedding in paraffin and sectioning. Sections were deparaffinized in xylene, followed by a graded series of alcohol washes. To determine the extent of cartilage deterioration, sections were stained with Safranin O/Fast Green (MilliporeSigma), and the histological scoring method issued by OARSI was used for analysis as described previously $(47,71,72)$. To determine degree of synovitis, joint sections were stained with Masson's trichrome stain and scored on a scale of $0-3$ ( 0 , no synovitis; 1 , mild synovitis; 2 , moderate synovitis; and 3 , severe synovitis) (47). Subchondral bone plate histomorphometry was performed on Safranin O/Fast Green-stained sections of knee joints of control and Wnt inhibitor-treated groups. Blinded histomorphometric assessment was performed on the medial compartment as described previously (49). Immunohistochemical staining for Ki-67 (Cell Signaling, 12202S), F480 (Novus Biologicals, NB600-404), CD68 (Leica, PA0191), and aSMA (ThermoFisher Scientific, RB-9010-P1) was performed by the Vanderbilt Translational Pathology Shared Resource. Briefly, the Leica Bond Max IHC stainer was used for deparaffinization, heat-induced antigen retrieval was performed with Epitope Retrieval 2 solution (Leica), blocking was performed with Protein Block (DAKO, 0909), and primary antibody incubation and visualization were performed with the Bond Refine Polymer detection system. Slides were then dehydrated, cleared, and mounted. Two independent readers evaluated slides in a blinded fashion. 
Plasma sample processing. Plasma was isolated from the saphenous vein of 10-week-old male C57BL/6J mice before injection with saline or XAV-939, 3 and 24 hours after injection. Plasma was stored at $-80^{\circ} \mathrm{C}$ in $2-\mathrm{ml}$ polypropylene tubes and allowed to thaw on ice before processing. Aliquots of thawed plasma (25 $\mathrm{ml}$ ) were transferred to clean microcentrifuge tubes and spiked with the internal standard carbamazepine $(2.5 \mathrm{ml})$. Calibration standards $(25 \mathrm{ml})$ were spiked with internal standard and the appropriate working stocks of XAV-939 $(2.5 \mathrm{ml})$. Spiked samples were lightly vortexed, allowed to stand at room temperature for 15-20 minutes, and deproteinized with $75 \mathrm{ml}$ cold acetonitrile. Precipitated proteins were removed by centrifugation $\left(18,000 \mathrm{~g}, 30\right.$ minutes, $\left.5^{\circ} \mathrm{C}\right)$. The clear supernatant $(\sim 75 \mathrm{ml})$ of each sample was transferred to a clean Eppendorf tube and evaporated under a gentle stream of $\mathrm{N}_{2}$ gas. The residue was reconstituted in $75 \mathrm{ml} \mathrm{H} \mathrm{O}_{2} / \mathrm{CH}_{3} \mathrm{OH}$ (3:1) containing $0.15 \%$ acetic acid, vigorously vortexed, centrifuged to remove particulates $\left(18,000 \mathrm{~g}, 30\right.$ minutes, $\left.5^{\circ} \mathrm{C}\right)$, and transferred to autosampler vials equipped with $200 \mathrm{ml}$ polypropylene inserts and Teflon-lined bonded rubber septa.

Nanoflow liquid chromatography/mass spectrometry analysis. XAV-939 was diluted in wild-type mouse plasma to generate a standard curve. Quantification of XAV-939 was based on single reaction monitoring (SRM) detection using an Orbitrap mass spectrometer (ThermoFisher Scientific). The mass spectrometer was operated in positive ion mode using the following optimized SRM transitions: XAV-939, $\mathrm{m} / z$ 313.1 $\rightarrow$ 267; CID CE 27\%, isolation width $2.0 \mathrm{~m} / z$; carbamazepine, $\mathrm{m} / z 237.1 \rightarrow 194$; CID CE 27\%, isolation width $2.0 \mathrm{~m} / z$. The following optimized parameters were used for the detection of analyte and internal standard: spray voltage, $2.3 \mathrm{kV}$; capillary temperature, $200^{\circ} \mathrm{C}$; capillary offset, $3 \mathrm{~V}$; tube lens offset, $80 \mathrm{~V}$; 3 microscans, AGC maximum inject time, $50 \mathrm{~ms}$. The instrument was calibrated weekly over a mass range of $\mathrm{m} / z 195-1,722$ (caffeine, MRFA, and Ultramark 1621) using the manufacturer's calibration procedure. All chromatographic separations were performed using an Easy-nLC 1000 (ThermoFisher Scientific) nanoflow LC system. Mobile phases were made up of $0.1 \% \mathrm{HCOOH}$ in (A) $\mathrm{H}_{2} \mathrm{O}$ and in (B) $\mathrm{CH}_{3} \mathrm{CN}$. Gradient conditions were as follows: $0-2$ minutes, $\mathrm{B}=15 \%$; $2-27$ minutes, $\mathrm{B}=15-100 \% ; 27-32$ minutes, $\mathrm{B}=100 \%$; $32-33.5$ minutes, $\mathrm{B}=100-15 \% ; 33.5-46.5$ minutes, $\mathrm{B}=15 \%$. The flow rate was maintained at $500 \mathrm{nl} / \mathrm{min}$. The autosampler injection valve and syringe needle were flushed sequentially with mobile phase $\mathrm{B}(2 \times 20 \mathrm{ml})$ and mobile phase $\mathrm{A}(2 \times 20 \mathrm{ml})$ between sample injections. The autosampler tray temperature was maintained at $10^{\circ} \mathrm{C}$. The analytical column was preequilibrated with $4 \mathrm{ml}$ mobile phase A at a constant back pressure of 200 bar prior to each injection cycle. Plasma extracts $(1 \mathrm{ml})$ were loaded at a constant back pressure of $200 \mathrm{bar}(6 \mathrm{ml}$ loading volume) onto a polyimide-coated, fused-silica capillary column (360 mm OD $\times 100 \mathrm{~mm}$ ID, Polymicro Technologies) packed with $15 \mathrm{~cm}$ Aqua-C18 bulk packing material (Phenomenex). Emitter tips ( $2 \mathrm{~mm}$ ) were pulled using a P-2000 Laser Based Micropipette Puller (Sutter Instrument). The Aqua C18 material was suspended in methanol, and columns were slurry packed to a desired capacity on a PicoView pressure loader (New Objective, Scientific Instruments Services) under 1,000 psi of ultra-high purity helium gas.

Data acquisition and processing. Data acquisition and quantitative spectral analysis were performed using Thermo-Finnigan Xcalibur version 2.0.7 and Thermo-Finnigan LCQuan version 2.5.6, respectively. Calibration curves were constructed by plotting the peak area ratio (XAV-939/carbamazepine) against the concentration of XAV-939 for a series of 6 plasma standards (20 pM-100 nM). A weighting factor of 1/ $\mathrm{C}^{2}$ was applied in the linear least-squares regression analysis to maintain homogeneity of variance across the concentration range. The lower limit of quantitation was $100 \mathrm{pM}$, defined as the lowest standard on the calibration curve, where $\% \mathrm{RE} \leq 20 \%$. The lower limit of detection was approximately $20 \mathrm{pM}$.

Cell culture. Human OA synovial tissues were obtained from patients that underwent knee joint replacement surgery under informed written consent. Control human synovial tissues were obtained from donors without a history of joint disease and with macroscopically healthy joints either at necropsy or knee arthroscopic surgery for minor meniscal tears under informed written consent. OA was diagnosed in all patients according to the American College of Rheumatology Diagnostic Criteria for OA (73). Synovial fibroblasts were prepared as previously described (74). Human chondrocytes were released from articular cartilage isolated from the femoral condyles by sequential enzymatic digestion as previously described (75). To preserve the chondrocyte phenotype, only first-passage cultured chondrocytes were used for cell culture experiments.

Chondrocytes and fibroblasts were serum starved in DMEM + 0\% FBS for 4 hours and treated with WNT3A (50 ng/ $\mu$ l) (Vanderbilt Antibody and Protein Resource) in DMEM + 2\% FBS for 24 hours; then, they were subjected to no treatment, C113 $(2 \mu \mathrm{M})$, or XAV-939 $(10 \mu \mathrm{M})$ for 24 hours and collected for protein or RNA extraction. The small-molecule Wnt inhibitor C113 was a gift from Ethan Lee, Department of Cell and Developmental Biology, Vanderbilt University (Nashville, Tennessee, USA) and was synthesized 
by the Vanderbilt Institute of Chemical Biology's medicinal chemistry core (31). XAV-939 was purchased from Selleck Chemicals (S1180) (30).

For conditioned media experiments, synovial fibroblasts were treated as above with WNT3A and inhibitors. After treatment, media were changed to serum-free DMEM for 24 hours and then collected. These media were either used for protein arrays (RayBiotech, Cytokine Array C5) or to treat chondrocytes for 24 hours.

To measure the effect of Wnt inhibition with XAV-939 and C113, NIH3T3 cells that overexpress $W n t 3 A$ were seeded overnight and then treated with XAV-939 and C113 over 24 hours. At timed intervals, the RNA levels of Axin2 were assessed by qRT-PCR. The media were then changed and cells were collected every 24 hours to assess reexpression of Axin2.

For apoptosis of chondrocytes, cells were treated with $80 \mathrm{mM}$ ethanol in conditioned medium for 24 hours in 8-well chamber slides and fixed with $4 \%$ paraformaldehyde.

Real-time RT-PCR. Quantitative real-time RT-PCR was used to quantify mRNA expression of AXIN2, COL1A1, COL2A1, PRG4, and MMP13 (Supplemental Table 2). Total RNA was isolated using TRIzol (Invitrogen). cDNA was generated using the iScript cDNA Synthesis kit (Bio-Rad), and RT-PCR was performed as described previously (76).

Immunoblotting. Total cell lysates were collected by suspension of cell pellets in RIPA buffer. Cell fractionation was performed with the NE-PER nuclear and cytoplasmic extraction kit per manufacturer's directions (ThermoFisher Scientific, catalog 78833). Protein was denatured and resolved by SDS-PAGE electrophoresis and transferred onto a nitrocellulose membrane (PerkinElmer). Blots were blocked in 5\% milk with TBST and then probed with COL1A1 (MDBioproducts, 203002), $\beta$-catenin (BD Transduction Laboratories, 610153), or $\beta$-actin (MilliporeSigma, A5441). Secondary antibodies were conjugated to HRP (goat anti-mouse (SouthernBiotech, 1010-05) or goat anti-rabbit (SouthernBiotech, 4050-05), and bands were visualized by chemiluminescence.

Immunofluorescence. Cells were seeded overnight in chamber slides and then fixed with acetone. Tissue sections were deparaffinized in xylene, followed by a graded series of alcohol washes. Antigen retrieval was performed by boiling in citrate buffer. Tissue sections were blocked with $10 \%$ goat serum; incubated with antibodies against COL1A1 (MDBioproducts, 203002), COL2A1 (Millipore, MAB8887), Vimentin (ThermoFisher Scientific, PA1-10003), active Jnk (Promega, V7931), Periostin (Santa Cruz, sc-67233), $\beta$-catenin (BD Transduction Laboratories, 610153), PCNA (Santa Cruz, sc-56), or SOX9 (Millipore, AB5535); incubated with secondary antibody (goat anti-mouse FITC (Leinco Technologies, M359) or goat anti-rabbit Cy3 (Jackson ImmunoResearch 111-165-144); and mounted with ProLong Gold antifade reagent with DAPI (Invitrogen). Isotype controls to rabbit (ThermoFisher Scientific, 02-6102) or mouse IgG (BioLegend, 400223) were included as appropriate with the same conditions and secondary antibodies. For TUNEL staining, a 1:10 mix of enzyme/label was added to the samples (In Situ Cell Death Detection Kit TMR Red, Roche) and then mounted with ProLong Gold Antifade reagent with DAPI (Invitrogen). Slides were viewed with a confocal laser scanning microscope (Zeiss LSM510).

Proliferation assay. Proliferation was assessed using the BrdU Cell Proliferation Assay kit (Calbiochem, EMD Millipore Corporation). Briefly, cells were seeded with or without WNT3A $(50 \mathrm{ng} / \mathrm{ml})$ for 24 hours. A subset of cells were treated with either C113 (1 mM) or XAV-939 (2 mM) for an additional 24 hours. The amount of BrdU incorporated into cultured cells was quantified calorimetrically using a spectrophotometer.

Statistics. The statistical significance was determined by 1-way or 2-way ANOVA with Tukey correction for multiple comparisons using multiple groups, as indicated in the figure legends. For comparison between two groups of data, unpaired 2-tailed $t$ test was used for normally distributed data sets with Welch's correction. GraphPad Prism software was used for all statistical analyses. $P<0.05$ was considered statistically significant.

Study approval. All animal studies were approved by the Animal Resources Centre, University Health Network. This study and collection of human-derived synovial fibroblasts and chondrocytes were approved by the University Health Network Institutional Ethics Committee Board (REB: 14-7529AE) and the Vanderbilt Institutional Review Board (IRB 131308).

\section{Author contributions}

$\mathrm{CL}$ and BW were involved in the conception and design of the study, acquisition of data, and analysis and interpretation of data; drafted the manuscript, revised it critically for important intellectual content, and approved the final version. SL was involved in acquisition of data and analysis and interpretation of data, drafted the manuscript, revised it critically for important intellectual content, and approved the final 
version. AS was involved in obtaining critical human specimen collections, drafted the manuscript, revised it critically for important intellectual content, and approved the final version. ER and MS prepared samples for histology, performed histopathological assessments, drafted the manuscript, revised it critically for important intellectual content, and approved the final version. PD and AS performed animal experiments and surgeries, drafted the manuscript, revised it critically for important intellectual content, and approved the final version. RG was involved in human specimen collection and characterization, drafted the manuscript, revised it critically for important intellectual content, and approved the final version. MK and PPY were involved in the conception and design of the study and interpretation of data; drafted the manuscript; revised it critically for important intellectual content; and approved the final version.

\section{Acknowledgments}

The authors would like to thank Lester Watch for his help with immunofluorescence of tissue sections. This work was supported by VA merit award 1BX002337A (to PPY), NIH grants R21EB019509, 1R01 GM118300-01 (to PPY), and The Campaign to Cure Arthritis via the Toronto General and Western Hospital Foundation (University Health Network) grant (to MK).

Address correspondence to: Pampee P. Young or Mohit Kapoor, 1161 21st Avenue South, MCN C2217, Nashville, Tennessee 37232, USA. Phone: 615.717 .7488 (P.P. Young); 416.603 .5800 ext. 4796 (M. Kapoor). Email: pampee.young@redcross.org (P.P. Young); Mohit.Kapoor@uhnresearch.ca (M. Kapoor).

1. Chen D, et al. Osteoarthritis: toward a comprehensive understanding of pathological mechanism. Bone Res. 2017;5:16044.

2. Scanzello CR, Goldring SR. The role of synovitis in osteoarthritis pathogenesis. Bone. 2012;51(2):249-257.

3. Bitton R. The economic burden of osteoarthritis. Am J Manag Care. 2009;15(8 Suppl):S230-S235.

4. Holt HL, et al. Forecasting the burden of advanced knee osteoarthritis over a 10 -year period in a cohort of $60-64$ year-old US adults. Osteoarthr Cartil. 2011;19(1):44-50.

5. Clevers H, Nusse R. Wnt/ $\beta$-catenin signaling and disease. Cell. 2012;149(6):1192-1205.

6. Day TF, Guo X, Garrett-Beal L, Yang Y. Wnt/beta-catenin signaling in mesenchymal progenitors controls osteoblast and chondrocyte differentiation during vertebrate skeletogenesis. Dev Cell. 2005;8(5):739-750.

7. Komiya Y, Habas R. Wnt signal transduction pathways. Organogenesis. 2008;4(2):68-75.

8. Nusse R. Wnt signaling in disease and in development. Cell Res. 2005;15(1):28-32.

9. Miller JR, Hocking AM, Brown JD, Moon RT. Mechanism and function of signal transduction by the Wnt/beta-catenin and Wnt/Ca2+ pathways. Oncogene. 1999;18(55):7860-7872.

10. Kurimoto $\mathrm{S}$, et al. Activation of the Wnt/ $\beta$-catenin signaling cascade after traumatic nerve injury. Neuroscience. 2015;294:101-108.

11. Xiao L, et al. Sustained activation of Wnt/ $\beta$-catenin signaling drives AKI to CKD progression. J Am Soc Nephrol. 2016;27(6):1727-1740

12. Surendran K, Schiavi S, Hruska KA. Wnt-dependent beta-catenin signaling is activated after unilateral ureteral obstruction, and recombinant secreted frizzled-related protein 4 alters the progression of renal fibrosis. J Am Soc Nephrol. 2005;16(8):2373-2384.

13. Tan RJ, Zhou D, Zhou L, Liu Y. Wnt/ß-catenin signaling and kidney fibrosis. Kidney Int Suppl (2011). 2014;4(1):84-90.

14. Bastakoty D, Saraswati S, Cates J, Lee E, Nanney LB, Young PP. Inhibition of Wnt/ $\beta$-catenin pathway promotes regenerative repair of cutaneous and cartilage injury. FASEB J. 2015;29(12):4881-4892.

15. Bastakoty D, et al. Temporary, systemic inhibition of the WNT/ $\beta$-catenin pathway promotes regenerative cardiac repair following myocardial infarct. Cell Stem Cells Regen Med. 2016;2(2):10.16966/2472-6990.111.

16. Dell'Accio F, et al. Activation of WNT and BMP signaling in adult human articular cartilage following mechanical injury. Arthritis Res Ther. 2006;8(5):R139.

17. Dell'accio F, De Bari C, Eltawil NM, Vanhummelen P, Pitzalis C. Identification of the molecular response of articular cartilage to injury, by microarray screening: Wnt-16 expression and signaling after injury and in osteoarthritis. Arthritis Rheum. 2008;58(5):1410-1421.

18. Yuasa T, Otani T, Koike T, Iwamoto M, Enomoto-Iwamoto M. Wnt/beta-catenin signaling stimulates matrix catabolic genes and activity in articular chondrocytes: its possible role in joint degeneration. Lab Invest. 2008;88(3):264-274.

19. Zhu M, et al. Activation of beta-catenin signaling in articular chondrocytes leads to osteoarthritis-like phenotype in adult beta-catenin conditional activation mice. J Bone Miner Res. 2009;24(1):12-21.

20. Corr M. Wnt-beta-catenin signaling in the pathogenesis of osteoarthritis. Nat Clin Pract Rheumatol. 2008;4(10):550-556.

21. Zhu M, et al. Inhibition of beta-catenin signaling in articular chondrocytes results in articular cartilage destruction. Arthritis Rheum. 2008;58(7):2053-2064

22. van den Bosch $\mathrm{MH}$, et al. Induction of canonical Wnt signaling by synovial overexpression of selected Wnts leads to protease activity and early osteoarthritis-like cartilage damage. Am J Pathol. 2015;185(7):1970-1980.

23. Oh H, Chun $\mathrm{CH}$, Chun JS. Dkk-1 expression in chondrocytes inhibits experimental osteoarthritic cartilage destruction in mice. Arthritis Rheum. 2012;64(8):2568-2578

24. Funck-Brentano T, Bouaziz W, Marty C, Geoffroy V, Hay E, Cohen-Solal M. Dkk-1-mediated inhibition of Wnt signaling in 
bone ameliorates osteoarthritis in mice. Arthritis Rheumatol. 2014;66(11):3028-3039.

25. Blom AB, van Lent PL, van der Kraan PM, van den Berg WB. To seek shelter from the WNT in osteoarthritis? WNT-signaling as a target for osteoarthritis therapy. Curr Drug Targets. 2010;11(5):620-629.

26. Bastakoty D, Young PP. Wnt/ $\beta$-catenin pathway in tissue injury: roles in pathology and therapeutic opportunities for regeneration. FASEB J. 2016;30(10):3271-3284.

27. Liu W, et al. Mutations in AXIN2 cause colorectal cancer with defective mismatch repair by activating beta-catenin/TCF signalling. Nat Genet. 2000;26(2):146-147.

28. Kinzler KW, Vogelstein B. Lessons from hereditary colorectal cancer. Cell. 1996;87(2):159-170.

29. Morin PJ, et al. Activation of beta-catenin-Tcf signaling in colon cancer by mutations in beta-catenin or APC. Science. 1997;275(5307):1787-1790.

30. Huang SM, et al. Tankyrase inhibition stabilizes axin and antagonizes Wnt signalling. Nature. 2009;461(7264):614-620.

31. Thorne CA, et al. Small-molecule inhibition of Wnt signaling through activation of casein kinase $1 \alpha$. Nat Chem Biol. 2010;6(11):829-836.

32. Li B, et al. Pyrvinium attenuates Hedgehog signaling downstream of smoothened. Cancer Res. 2014;74(17):4811-4821.

33. Li B, et al. Differential abundance of CK1 $\alpha$ provides selectivity for pharmacological CK1 $\alpha$ activators to target WNT-dependent tumors. Sci Signal. 2017;10(485):eaak9916.

34. Saraswati S, Alfaro MP, Thorne CA, Atkinson J, Lee E, Young PP. Pyrvinium, a potent small molecule Wnt inhibitor, promotes wound repair and post-MI cardiac remodeling. PLoS ONE. 2010;5(11):e15521.

35. Chen L, Wu Q, Guo F, Xia B, Zuo J. Expression of Dishevelled-1 in wound healing after acute myocardial infarction: possible involvement in myofibroblast proliferation and migration. J Cell Mol Med. 2004;8(2):257-264.

36. Chen Y, et al. Beta-catenin signaling plays a disparate role in different phases of fracture repair: implications for therapy to improve bone healing. PLoS Med. 2007;4(7):e249

37. Fernández-Martos CM, González-Fernández C, González P, Maqueda A, Arenas E, Rodríguez FJ. Differential expression of Wnts after spinal cord contusion injury in adult rats. PLoS One. 2011;6(11):e27000.

38. Königshoff M, et al. WNT1-inducible signaling protein-1 mediates pulmonary fibrosis in mice and is upregulated in humans with idiopathic pulmonary fibrosis. J Clin Invest. 2009;119(4):772-787.

39. Lories RJ, Corr M, Lane NE. To Wnt or not to Wnt: the bone and joint health dilemma. Nat Rev Rheumatol. 2013;9(6):328-339

40. Ma B, van Blitterswijk CA, Karperien M. A Wnt/ $\beta$-catenin negative feedback loop inhibits interleukin-1-induced matrix metalloproteinase expression in human articular chondrocytes. Arthritis Rheum. 2012;64(8):2589-2600.

41. Landman EB, Miclea RL, Van Blitterswijk CA, Karperien M. Small molecule inhibitors of Wnt/b-catenin signaling block IL-1b and TNF-a induced cartilage degradation. Arthritis Res Ther. 2013;15(4):R93.

42. Takamatsu A, et al. Verapamil protects against cartilage degradation in osteoarthritis by inhibiting Wnt/ $\beta$-catenin signaling PLoS One. 2014;9(3):e92699.

43. Shi S, Man Z, Li W, Sun S, Zhang W. Silencing of Wnt5a prevents interleukin-1 $\beta$-induced collagen type II degradation in rat chondrocytes. Exp Ther Med. 2016;12(5):3161-3166.

44. Glasson SS, Blanchet TJ, Morris EA. The surgical destabilization of the medial meniscus (DMM) model of osteoarthritis in the 129/SvEv mouse. Osteoarthr Cartil. 2007;15(9):1061-1069.

45. Jho EH, Zhang T, Domon C, Joo CK, Freund JN, Costantini F. Wnt/beta-catenin/Tcf signaling induces the transcription of Axin2, a negative regulator of the signaling pathway. Mol Cell Biol. 2002;22(4):1172-1183.

46. Thorne CA, et al. Small-molecule inhibition of Wnt signaling through activation of casein kinase $1 \alpha$. Nat Chem Biol. 2010;6(11):829-836.

47. Glasson SS, Chambers MG, Van Den Berg WB, Little CB. The OARSI histopathology initiative - recommendations for histological assessments of osteoarthritis in the mouse. Osteoarthr Cartil. 2010;18 Suppl 3:S17-S23.

48. Krenn V, Morawietz L, Häupl T, Neidel J, Petersen I, König A. Grading of chronic synovitis--a histopathological grading system for molecular and diagnostic pathology. Pathol Res Pract. 2002;198(5):317-325.

49. Valverde-Franco G, et al. In vivo bone-specific EphB4 overexpression in mice protects both subchondral bone and cartilage during osteoarthritis. Arthritis Rheum. 2012;64(11):3614-3625.

50. Bhattaram P, Chandrasekharan U. The joint synovium: A critical determinant of articular cartilage fate in inflammatory joint diseases. Semin Cell Dev Biol. 2017;62:86-93.

51. Lacey D, et al. Control of fibroblast-like synoviocyte proliferation by macrophage migration inhibitory factor. Arthritis Rheum. 2003;48(1):103-109.

52. Qu Z, Garcia CH, O'Rourke LM, Planck SR, Kohli M, Rosenbaum JT. Local proliferation of fibroblast-like synoviocytes contributes to synovial hyperplasia. Results of proliferating cell nuclear antigen/cyclin, c-myc, and nucleolar organizer region staining. Arthritis Rheum. 1994;37(2):212-220.

53. Remst DF, et al. Gene expression analysis of murine and human osteoarthritis synovium reveals elevation of transforming growth factor beta-responsive genes in osteoarthritis-related fibrosis. Arthritis Rheumatol. 2014;66(3):647-656.

54. Alquraini A, et al. The interaction of lubricin/proteoglycan 4 (PRG4) with toll-like receptors 2 and 4: an anti-inflammatory role of PRG4 in synovial fluid. Arthritis Res Ther. 2015;17:353.

55. Waller KA, et al. Intra-articular recombinant human proteoglycan 4 mitigates cartilage damage after destabilization of the medial meniscus in the Yucatan minipig. Am J Sports Med. 2017;45(7):1512-1521.

56. Heathfield TF, Onnerfjord P, Heinegard D, Dahlberg L. Proteolytic removal of the N-terminal region of fibromodulin in IL-1 stimulated bovine cartilage explants. Arthritis Rheum. 2001;44(9):S43.

57. Jia H, et al. EGFR signaling is critical for maintaining the superficial layer of articular cartilage and preventing osteoarthritis initiation. Proc Natl Acad Sci USA. 2016;113(50):14360-14365.

58. Kahn M. Can we safely target the WNT pathway? Nat Rev Drug Discov. 2014;13(7):513-532.

59. Nalesso G, et al. WNT16 antagonises excessive canonical WNT activation and protects cartilage in osteoarthritis. Ann Rheum Dis. 2017;76(1):218-226.

60. Usami Y, Gunawardena AT, Iwamoto M, Enomoto-Iwamoto M. Wnt signaling in cartilage development and diseases: lessons 
from animal studies. Lab Invest. 2016;96(2):186-196.

61. Loughlin J, et al. Functional variants within the secreted frizzled-related protein 3 gene are associated with hip osteoarthritis in females. Proc Natl Acad Sci USA. 2004;101(26):9757-9762

62. Diarra D, et al. Dickkopf-1 is a master regulator of joint remodeling. Nat Med. 2007;13(2):156-163.

63. van den Bosch MH, et al. Synovial Wnt signaling induces the expression of MMPs is associated with disease progression in early symptomatic osteoarthritis patients. Arthritis Rheumatol. 2017;69(10):1978-1983.

64. Chen L, Wu Y, Wu Y, Wang Y, Sun L, Li F. The inhibition of EZH2 ameliorates osteoarthritis development through the Wnt/ $\beta$-catenin pathway. Sci Rep. 2016;6:29176.

65. Hood JD, Deshmukh V, Barroga C, Hu Y. OP0069 Discovery of a small molecule inhibitor of the WNT pathway (SM04690) as a potential disease modifying treatment for knee osteoarthritis. Ann Rheum Dis. 2016;75:81.

66. Tchetina EV, Squires G, Poole AR. Increased type II collagen degradation and very early focal cartilage degeneration is associated with upregulation of chondrocyte differentiation related genes in early human articular cartilage lesions. J Rheumatol. 2005;32(5):876-886.

67. Musumeci G, et al. Lubricin expression in human osteoarthritic knee meniscus and synovial fluid: a morphological, immunohistochemical and biochemical study. Acta Histochem. 2014;116(5):965-972.

68. Loeser RF. Osteoarthritis year in review 2013: biology. Osteoarthr Cartil. 2013;21(10):1436-1442.

69. Ruan MZ, et al. Proteoglycan 4 expression protects against the development of osteoarthritis. Sci Transl Med. 2013;5(176):176ra34.

70. Benito MJ, Veale DJ, FitzGerald O, van den Berg WB, Bresnihan B. Synovial tissue inflammation in early and late osteoarthritis. Ann Rheum Dis. 2005;64(9):1263-1267.

71. Vasheghani F, et al. PPAR $\gamma$ deficiency results in severe, accelerated osteoarthritis associated with aberrant mTOR signalling in the articular cartilage. Ann Rheum Dis. 2015;74(3):569-578.

72. Zhang Y, et al. Cartilage-specific deletion of mTOR upregulates autophagy and protects mice from osteoarthritis. Ann Rheum Dis. 2015;74(7):1432-1440.

73. Altman R, et al. Development of criteria for the classification and reporting of osteoarthritis. Classification of osteoarthritis of the knee. Diagnostic and Therapeutic Criteria Committee of the American Rheumatism Association. Arthritis Rheum. 1986;29(8):1039-1049.

74. Nakamachi Y, et al. Specific increase in enzymatic activity of adenosine deaminase 1 in rheumatoid synovial fibroblasts. Arthritis Rheum. 2003;48(3):668-674.

75. Martel-Pelletier J, Mineau F, Jovanovic D, Di Battista JA, Pelletier JP. Mitogen-activated protein kinase and nuclear factor kappaB together regulate interleukin-17-induced nitric oxide production in human osteoarthritic chondrocytes: possible role of transactivating factor mitogen-activated protein kinase-activated protein kinase (MAPKAPK). Arthritis Rheum. 1999;42(11):2399-2409

76. Segedy AK, et al. Identification of small proline-rich repeat protein 3 as a novel atheroprotective factor that promotes adaptive Akt signaling in vascular smooth muscle cells. Arterioscler Thromb Vasc Biol. 2014;34(12):2527-2536. 